\title{
Computational Study on Three-Dimensional Convective Casson Nanofluid Flow past a Stretching Sheet with Arrhenius Activation Energy and Exponential Heat Source Effects
}

\author{
P. Ragupathi $\mathbb{i}^{1},{ }^{1}$ S. Saranya ${ }^{(D},{ }^{2}$ H.V.R. Mittal ${ }^{(D)},{ }^{3}$ and Qasem M. Al-Mdallal ${ }^{2}{ }^{2}$ \\ ${ }^{1}$ Department of Mathematics, Sri Ramakrishna Mission Vidyalaya College of Arts and Science, Coimbatore-641 020, India \\ ${ }^{2}$ Department of Mathematical Sciences, UAE University, P.O. Box 15551, Al-Ain, UAE \\ ${ }^{3}$ Computer, Electrical and Mathematical Sciences and Engineering Division, \\ King Abdullah University of Science and Technology, (KAUST), Thuwal 23955-6900, Saudi Arabia
}

Correspondence should be addressed to Qasem M. Al-Mdallal; q.almdallal@uaeu.ac.ae

Received 13 June 2021; Accepted 21 August 2021; Published 30 November 2021

Academic Editor: Ali Akgül

Copyright (C) 2021 P. Ragupathi et al. This is an open access article distributed under the Creative Commons Attribution License, which permits unrestricted use, distribution, and reproduction in any medium, provided the original work is properly cited.

\begin{abstract}
The effective applications of Casson fluid in drilling processes, biological treatments, food processing, and bio-engineering activities have caught the interest of a wide range of researchers. The suitable knowledge of heat transfer via non-Newtonian fluid is essential for the achievement of best quality products in industry. Thus, the three-dimensional Casson nanofluid flow over a stretching sheet with Arrhenius activation energy and exponential heat source effects is investigated in this paper using a computational process based on iterative power series (IPS) method. To provide useful insights into the physical and dynamic examinations of this topic, convective heat and convective mass boundary conditions are used. The developed model of nonlinear partial differential equations (PDEs) has been transformed into ordinary differential equations (ODEs) using similarity transformations. The numerical solution of the transformed ODEs is obtained by employing the IPS technique combined with shooting iteration approach. The results of this study are validated with the previous studies, and excellent agreements have been obtained. The behavior of various capable physical parameters is analyzed. It is observed that the thermal and concentration fields show an enhancement with respect to the exponential heat source parameter and thermal and concentration Biot numbers. Further, the Arrhenius activation energy parameter has shown a significant effect on the concentration field.
\end{abstract}

\section{Introduction}

A better understanding of engineering and industrial technology difficulties requires taking into account the boundary layer phenomenon of non-Newtonian liquids with heat transport. Non-Newtonian physiological liquids are used in various engineering processes such as manufacturing of fiberglass, paper, crystals and plastic sheets, food production, petroleum drilling, and transportation of slurries. Non-Newtonian fluids have a tendency to modify their viscosity or flow behavior under stress. Under the influence of an unexpected stress, certain non-Newtonian fluids such as Casson fluids, micropolar fluids, Carreau fluids, power law fluids, Prandtl fluids and Eyring-Powell fluids may behave similar to a solid due to an increase in their viscosity.
However, some situations might result in a reverse action where the fluid viscosity can decrease and fluids become thinner under the application of an unexpected stress. On the removal of these unexpected stresses, these liquids revert to their original flow behavior. Casson liquids have interesting properties and perform a significant part in nonNewtonian fluid experimentation, and in past few years they have gained the attention among researchers. The Casson fluid model was developed by Casson and Mill [1] for the prevision of pigment-oil suspension stream conduction. Shafiq et al. [2] analyzed the Darcy-Forchheimer Casson water/glycerin rotating nanofluid flow with uniform magnetic field. Saeed et al. [3] considered three-dimensional (3D) nano-Casson slender film stream created on a slanting rotating plate. Reddy et al. [4] analyzed the Buongiorno 
model with respect to the rotating flow of Casson liquids on an extended surface with thermophoresis and Brownian motion. The Casson nanofluid 3D stream over the permeable layers of a slender sheet in a graphene nanoparticulate suspension was investigated by Durgaprasad et al. [5]. Later, Durgaprasad et al. [6] studied the 3D slip stream of a chemically reactive Casson liquid flowing over a thin penetration layer with nonuniform heat source or sink. Raza [7] contemplated the impacts of radiation and slip on the magnetohydrodynamic stagnation point stream of Casson liquids past a convective stretching sheet. Thumma et al. [8] considered the impacts of the viscous dissipation and heat generation of nanofluid streams past an impermeable extending sheet. Murthy et al. [9] investigated the thermophoresis and Brownian movement effects in case of an exponentially extended surface in a 3D magnetohydrodynamic slip stream of the Casson nanofluid.

Investigations of non-Newtonian fluid flows created by a stretching sheet for MHD flow and heat transfer find many applications in engineering and industry such as melt spinning, extrusion, glass fiber processing, heat rolling, plastic wire drawing, rubber sheet manufacturing, and cooling of a solid steel plate in a pool. Sometimes sheets are continually stretched during manufacture to produce the necessary thickness. It demonstrates that the final outcome is determined by the sheet's stretching and cooling rate. For example, when molten polymers are extruded through a slit die to make plastic sheets, the sheet is stretched. In such operations, the pace of cooling determines the final product's qualities. When such a sheet is drawn in an electrically conducting Casson fluid under the influence of a magnetic field, the pace of cooling may be regulated, resulting in the desired final product. Crane [10] examined a Newtonian fluid boundary layer flow caused by the stretching elastic flat plate. He focused on the case of linear stretching elastic flat plate for discussing the heat conduction properties. Wang [11] analyzed a 3D flow past a stretching sheet. Freidoonimehr and Rahimi [12] studied the Brownian motion effect on the heat transfer of a 3D nanofluid flow past a stretched sheet with velocity slip. Hamid et al. [13] investigated the effects of linear thermal radiation and presented a dual solution for both steady and unsteady flows of the Casson fluid over the stretching sheet. Aziz and Afify [14] analyzed the Casson fluid flow and showed that the production of entropy increased with increasing magnetization. In addition, a reverse behavior was observed with the Hall effect. Shankar et al. [15] demonstrated the 3D flow in a Casson fluid considering the Cattaneo-Christov heat flux and the Joule effect at the boundary of the stretching sheet. Mahanta et al. [16] discussed the Brownian and thermophoresis flow effects on the Casson nanofluid over a stretching sheet in the presence of magnetic field.

Activation energy is defined as the minimum needed energy that reactants must receive to envision a chemical reaction. The activation energy has various focal points with respect to the design of chemical production, manufacturing of food items, geothermal repository, oil emulsion formation, etc. Activation energy investigations of a nano Casson fluid flow were carried out by Gireesha et al. [17] with an exponential spatial source of thermal energy and a binary chemical reaction. Hayat et al. [18] detailed the Arrhenius activation energy on the nanofluid 3D Darcy-Forchheimer stream based on the impacts of the binary chemical reaction in a rotating frame. Umar et al. [19] presented a numerical treatment of the 3D Eyring-Powell liquid stream over an expanding sheet with velocity slip and activation energy. Hayat et al. [20] introduced the properties of activation energy in the Carreau liquid stream with cross-dissemination and exponential heat source effects. Rashid et al. [21] introduced a 3D rotating Darcy-Forchheimer stream with activation energy. In the MHD third-grade nanofluid system, Hayat et al. [22] developed a computational model for studying the impact of the Arrhenius activation energy through a nonlinear extending surface under convective temperature and mass conditions. Rashid et al. [23] proposed a similar work with activation energy on an OldroydB nanofluid. Rashid et al. [24] also used Arrhenius energy for rotating the Maxwell nanomaterial. The activation energy in case of a squeezed liquid with a binary chemical reaction was examined by Ahmad et al. [25].

Researchers recently expressed their enthusiasm in the progress of methodologies, including heat generation/absorption due to its immense usage in the fields of information science and mechanical engineering (Nadeem et al. [26], Shehzad et al. [27], Mishra et al. [28], Rehman et al. [29], and Ragupathi et al. [30]). Except for the traditional component of the heat source, an irregular part of the heat source (thermal and exponential space-based source) is optimal for systems that require high-temperature distribution mechanisms. Mahanthesh et al. [31] examined the changes associated with radiative dusty nanofluid flow when subjected to the heat sources (exponential space-based) and Marangoni convection. Kumar et al. [32] considered exponential heat source effects on a fluid passing over a melting surface. Similarly, Sandeep et al. [33], Krishna et al. [34], Zia et al. [35], etc. contributed to establish the functioning of the heat source which is exponential space-based.

The nonlinear boundary value problems (BVPs) govern various phenomena in science and engineering. Accordingly, BVPs have therefore drawn great interest from researchers and technologists in obtaining and evaluating their solutions. BVPs are not trivial, and it is very difficult to find empirical solutions to nonlinear BVPs. The BVPs of real-life problems (fluid flow problems) are typically nonlinear and involve numerical and analytical algorithms with great accuracy in their solutions. Several numerical methods have been developed for solving such problems. The power series approach is perhaps the simplest and most effective approach compared with other nonlinear differential equation approaches (Liao and Tan [36]). This approach is frequently used on many problems including chaotic processes as almost a suitable mathematical approach. This approach has led to the development of a number of numerical methods and algorithms. An iterative method called the Iterative Power Series (IPS), based on recursive power series expansions, provides such a highly accurate numerical scheme (Burden and Faires [37]). The IPS procedure aims to ensure high precision by showing that the radius convergence of the 
solution can be altered to any major value by accuracy. In theory, this value reaches infinity, which produces exact solutions. The IPS solutions to $\operatorname{sech}(x)$ and nonlinear differential equations were presented by Al-Khawaja and AlMdallal [38]. The numerical IPS method was applied to study the fluid stream past a shrinking infinite permeable long cylinder by Al-Sakkaf et al. [39]. Their observations reported that the iterative numerical scheme obtained via this process is more useful than conventional numerical systems.

Based on the detailed literature survey, no study has investigated the $3 \mathrm{D}$ convective Casson nanofluid flow with Arrhenius activation energy and exponential heat source effects past a stretching sheet. This analysis therefore incorporates some new aspects, which are described hereinafter.

(i) Convective heat and mass boundary conditions are added to explore and enhance the dynamical investigations.

(ii) A notable shooting procedure and IPS method are employed to examine the influence of numerous parameters (magnetic, exponential heat source, and Arrhenius activation energy) on velocity, energy, and concentration profiles.

(iii) Present numerical results are compared with those obtained by Umar et al. [19], Freidoonimehr and Rahimi [12], and Wang [11]. They observed the movement of 3D fluid flow over a stretching layer, which was observed herein to exhibit perfect coordination.

(iv) The appearance of capable physical quantities with respect to standard profiles is visualized and analyzed.

The current study contributes to the literature by looking into the factors that influence the flow, heat, and mass transfer rate. Hence, the following research questions are central to the empirical part:

(i) Does the magnetic field and the sheets stretching rate increase the coefficient of skin friction?

(ii) Does the exponential heat source parameter influence the heat and mass transfer rate?

(iii) Does the thermal and concentration Biot numbers support the thermal and concentration profiles?

(iv) Does the Arrhenius activation energy supports thermal heat and mass transfer rate?

The remaining of the manuscript is organized in the following pattern. Section 2 gives the generalized formulation Casson model. Section 3 holds the description on Arrhenius activation equation and exponential heat source. Section 4 deals with the problem formulation, corresponding governing equations and boundary conditions. In
Section 5, the algorithm for IPS procedure and properties are explained. Section 6 explains the numerical algorithm of the IPS procedure followed by the validation of our numerical scheme in Section 7. Results and discussions are presented in Section 8, and finally the major conclusions are drawn in Section 9.

\section{Generalized Casson Fluid Model}

The rheological state for the Casson model is represented as [33]

$$
\tau^{*}=\tau_{0}+\mu \gamma^{*}
$$

or

$$
\tau_{i j}= \begin{cases}2\left(\frac{\sqrt{2 \prod} \mu_{B}+p_{y}}{\sqrt{2 \prod}}\right) e_{i j}, & \prod_{c}<\prod, \\ 2\left(\frac{\sqrt{2 \prod_{c}} \mu_{B}+p_{y}}{\sqrt{2 \prod_{c}}}\right) e_{i j}, & \prod>\prod,\end{cases}
$$

where $\Pi=e_{i j} e_{i j}$ is the product of the component of the deformation rate, $e_{i j}$ is the $(i, j)^{\text {th }}$ component of deformation rate, $\Pi_{c}$ is the critical value of this product, $\mu_{B}$ is the plastic dynamic viscosity of the fluid, and $p_{y}=\mu_{B} \sqrt{2 \Pi} / \beta$ is the yield stress of the fluid. To retain a steady strain rate, there are certain fluids that involve a progressive rise in shear stress and are known as rheopectic fluid. In case of Casson fluid where $\Pi>\Pi_{c}$ :

$$
\mu=\mu_{B}+\frac{p_{y}}{\sqrt{2 \Pi}}
$$

By substituting $p_{y}$ in equation (3), we can observe the dependency of kinematic viscosity $v$ of the Casson fluid on plastic dynamic viscosity $\mu_{B}$, density $\rho$, and Casson parameter $\beta$ as follows:

$$
v=\frac{\mu_{B}}{\rho}\left(1+\frac{1}{\beta}\right) .
$$

\section{Activation Energy and Exponential Heat Source}

3.1. The Arrhenius Equation. In 1889, Swedish scientist Svante Arrhenius discovered the presence of activation energy. To describe the relationship between temperature and reaction rate, Arrhenius devised his eponymous equation. The Arrhenius equation is essential for determining chemical reaction rates and, more critically, the amount of energy required to initiate them.

$$
K=A e^{-E_{a} / \mathrm{RT}},
$$


where $K$ is the reaction rate coefficient (the rate of reaction), $A$ is the frequency factor (how often molecules collide), $R$ is the universal gas constant, $T$ represents the absolute temperature, and $E_{a}$ is the activation energy.

3.2. Exponential Heat Source. Chemical or nuclear processes produce heat energy within a body as an interior energy source/sink. Its importance can be seen in combustion research, nuclear reactors, heat exchangers, plastic, paper, and steel production, thermal insulation, and fusion reactors, etc. The effects of an internal heat source/sink can be studied using two models. The first is a uniform temperature-dependent internal heat source/sink process $\left(Q_{0}\left(T-T_{\infty}\right)\right)$, and the second is a nonuniform space and a thermal based heat source in which two dimensionless parameters appear in the energy equation, one for thermal based heat source analysis and the other for space-based heat source analysis. These two models may not be able to achieve more heat transmission in the fluid boundary layer, according to science. An exponential heat source effect $\left(Q_{0}\left(T-T_{\infty}\right)\right.$ $e^{-m \sqrt{a / v} z}$ ) has been developed as a new model for intensive heating processes in light of these findings.

\section{Modeling and Description}

We consider the steady, laminar, and incompressible 3D flow of the Casson fluid past over a stretching sheet. The sheet is positioned at $z=0$, and the flow is assumed to occur in the domain $z>0$. Let the sheet's stretching velocity in $x$-direction be $u=U_{w}(x)=a x$ and the $y$-direction stretching velocity be $v=V_{w}(y)=b y$. Convective heat $\left(T_{f}\right)$ and mass $\left(C_{f}\right)$ conditions are imposed at the bottom of the sheet surface with coefficients $h_{f}$ and $h_{s}$. The schematic diagram of the physical domain of the problem is presented in Figure 1. The magnetic field is uniformly distributed with an impact force $B_{0}$ in a direction normal to the fluid flow. The induced magnetic field is neglected because of the small magnetic Reynolds number. The impacts of viscous dissipation are also neglected because the magnetic field is not considerably high. Attention is devoted to the novel Arrhenius activation energy and exponential heat source effects, which are comprehensively examined.

Based on all the above-stated assumptions, the following sequence of equations governing the flow is obtained.

$$
\begin{gathered}
\frac{\partial u}{\partial x}+\frac{\partial v}{\partial y}+\frac{\partial w}{\partial z}=0 \\
u \frac{\partial u}{\partial x}+v \frac{\partial u}{\partial y}+w \frac{\partial u}{\partial z}=v\left(\beta^{-1}+1\right) \frac{\partial^{2} u}{\partial z^{2}}-\frac{\sigma B_{0}^{2} u}{\rho} \\
u \frac{\partial v}{\partial x}+v \frac{\partial v}{\partial y}+w \frac{\partial v}{\partial z}=v\left(\beta^{-1}+1\right) \frac{\partial^{2} v}{\partial z^{2}}-\frac{\sigma B_{0}^{2} v}{\rho}, \\
u \frac{\partial T}{\partial x}+v \frac{\partial T}{\partial y}+w \frac{\partial T}{\partial z}=\alpha^{*} \frac{\partial^{2} T}{\partial z^{2}}+\tau\left[D_{B} \frac{\partial C}{\partial T} \frac{\partial T}{\partial z}+\frac{D_{T}}{T_{\infty}}\left(\frac{\partial T}{\partial z}\right)^{2}\right]+\frac{Q_{0}\left(T-T_{\infty}\right)}{\rho C_{p}} e^{-m \sqrt{a / v} z} \\
u \frac{\partial C}{\partial x}+v \frac{\partial C}{\partial y}+w \frac{\partial C}{\partial z}=D_{B}\left(\frac{\partial^{2} C}{\partial z^{2}}\right)+\frac{D_{T}}{T_{\infty}}\left(\frac{\partial^{2} T}{\partial z^{2}}\right)-K_{s}^{2}\left(C-C_{\infty}\right)\left(\frac{T}{T_{\infty}}\right)^{r} e^{\left(-E_{a} / k_{1} T\right)}
\end{gathered}
$$

where $u, v$, and $w$ are the $x, y$, and $z$ components of velocity, $\nu$ is the kinematic viscosity of the nanofluid, $\beta$ is the Casson parameter, $\rho$ is the density of the nanofluid, $T$ is the temperature of the fluid, $Q_{0}$ is the heat generation/absorption variable, and $D_{B}$ and $D_{T}$ represent the coefficient of
Brownian and thermophoresis diffusions, $K_{s}$ is the reaction rate, $E_{a}$ is the activation energy, and $k_{1}$ is the Boltzmann constant, respectively.

The corresponding boundary conditions are as follows:

$$
\begin{aligned}
& \left.\begin{array}{l}
u=U_{w}(x), v=V_{w}(y), w=0, \\
\frac{\partial T}{\partial z}=-\frac{h_{f}}{k_{f}}\left(T_{f}-T\right), \frac{\partial C}{\partial z}=-\frac{h_{s}}{D_{B}}\left(C_{f}-C\right)
\end{array}\right\} \text { at } z=0, \\
& \text { such that } u \longrightarrow 0, v \longrightarrow 0, w \longrightarrow 0, T \longrightarrow T_{\infty}, C \longrightarrow C_{\infty} \text { as } z \longrightarrow \infty .
\end{aligned}
$$




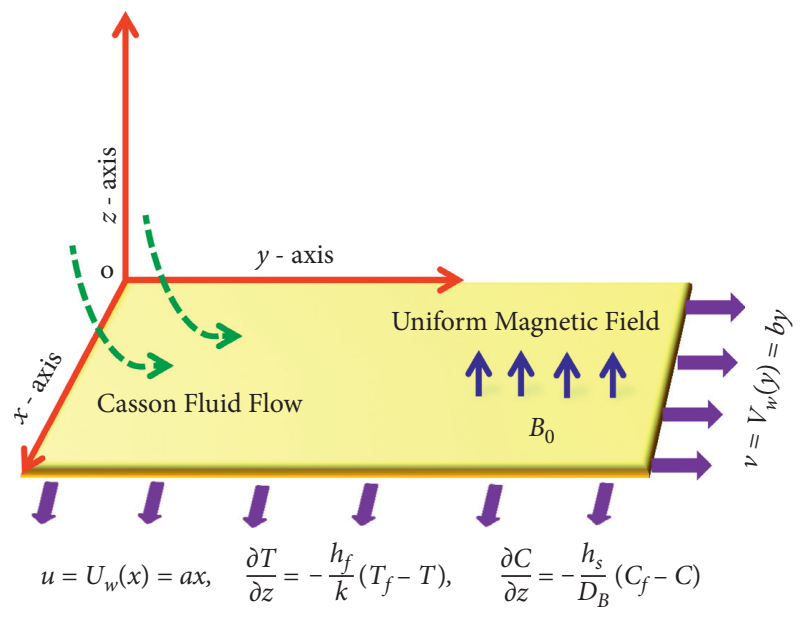

FIGURE 1: Schematic model of the physical coordinate system of the problem.

The variables for the similarity solution are given as follows:

$$
\begin{aligned}
\eta & =z \sqrt{\frac{a}{v}}, u=a x f^{\prime}(\eta), v=a y g^{\prime}(\eta), w \\
& =-\sqrt{a v}(f(\eta)+g(\eta)), \\
\theta(\eta) & =\frac{T-T_{\infty}}{T_{f}-T_{\infty}}, \phi(\eta)=\frac{C-C_{\infty}}{C_{f}-C_{\infty}} .
\end{aligned}
$$

By substituting the similarity variables from equation (12) into Equations (6)-(10), the transformed dimensionless equations can be written as

$$
\begin{gathered}
\left(\beta^{-1}+1\right) f^{\prime \prime \prime}+(f+g) f^{\prime \prime}-f^{\prime 2}-M f^{\prime}=0, \\
\left(\beta^{-1}+1\right) g^{\prime \prime \prime}+(f+g) g^{\prime \prime}-g^{\prime 2}-M g^{\prime}=0, \\
\theta^{\prime \prime}+\operatorname{Pr}(f+g) \theta^{\prime}+\operatorname{Pr} N b \theta^{\prime} \phi^{\prime}+\operatorname{Pr} N t \theta^{\prime 2}+\operatorname{PrQ} \theta e^{-m \eta}=0, \\
\phi^{\prime \prime}+L e(f+g) \phi^{\prime}+\frac{N t}{N b} \theta^{\prime \prime}-L e \Lambda(1+\Gamma \theta)^{r} e^{(-E / 1+\Gamma \theta)}=0 .
\end{gathered}
$$

After modifications, the boundary condition can be represented as follows:

$$
\begin{aligned}
& \left.\begin{array}{l}
f=0, g=0, f^{\prime}=1, g^{\prime}=\alpha, \\
\theta^{\prime}=-B i_{1}(1-\theta(0)), \phi^{\prime}=-B i_{2}(1-\phi(0))
\end{array}\right\} \text { at } \eta=0, \\
& f^{\prime} \longrightarrow 0, g^{\prime} \longrightarrow 0, \theta \longrightarrow 0, \phi \longrightarrow 0, \text { as } \eta \longrightarrow \infty .
\end{aligned}
$$

The nondimensional parameters used in equations (13)-(17) and their corresponding expressions are represented in Table 1.
TABLE 1: Nondimensional parameters and their expression.

\begin{tabular}{lc}
\hline Expression & Names \\
\hline$\alpha=b / a$ & Stretching ratio parameter \\
$M=\sigma B_{0}^{2} / \rho a$ & Magnetic parameter \\
$P r=v / \alpha$ & Prandtl number \\
$N b=\tau D_{B} / \nu\left(C_{f}-C_{\infty}\right)$ & Brownian motion parameter \\
$N t=\tau D_{T} / v T_{\infty}\left(T_{f}-T_{\infty}\right)$ & Thermophoresis parameter \\
$Q=Q_{0} / a \rho C_{p}$ & Exponential heat source parameter \\
$L e=v / D_{B}$ & Lewis number \\
$\Lambda=K_{s}^{2} / c$ & Reaction rate constant \\
$\Gamma=\left(T_{f}-T_{\infty}\right) / T_{\infty}$ & Nondimensional fluid parameter \\
$E=E a / k_{1} T_{\infty}$ & Arrhenius activation energy \\
$B i_{1}=h_{f} / k_{f} \sqrt{v / a}$ & parameter \\
$B i_{2}=h_{s} / D_{B} \sqrt{v / a}$ & Thermal biot number \\
\hline
\end{tabular}

The local skin friction coefficients, $C_{f x}$ and $C_{f y}$, the local Nusselt number, $\mathrm{Nus}_{x}$, and the local Sherwood number, $\mathrm{Sh}_{x}$, are given as

$$
\begin{aligned}
C_{f x} & =\frac{\tau_{w x}}{\rho U_{w}^{2}}, \\
C_{f y} & =\frac{\tau_{w y}}{\rho V_{w}^{2}}, \\
\mathrm{Nus}_{x} & =\frac{x q_{w}}{k\left(T_{f}-T_{\infty}\right)}, \\
\mathrm{Sh}_{x} & =\frac{x q_{m}}{D_{B}\left(C_{f}-C_{\infty}\right)},
\end{aligned}
$$

where $\tau_{w x}=\mu(\partial u / \partial z)_{z=0}$ and $\tau_{w y}=\mu(\partial v / \partial z)_{z=0}$ are the shear stress values along the surface of the sheet, $q_{w}=-k(\partial T / \partial z)_{z=0}$ is the heat flux, and $q_{m}=-D_{B}(\partial C / \partial z)_{z=0}$ is the mass flux.

The dimensionless form of equation (18) can be written as:

$$
\begin{aligned}
\operatorname{Re}_{x}^{1 / 2} C_{f x} & =\left(\beta^{-1}+1\right) f^{\prime \prime}(0), \\
\alpha^{3 / 2} \operatorname{Re}_{y}^{1 / 2} C_{f y} & =\left(\beta^{-1}+1\right) g^{\prime \prime}(0), \\
\operatorname{Re}_{x}^{-1 / 2} \mathrm{Nus}_{x} & =-\theta^{\prime}(0), \\
\operatorname{Re}_{x}^{-1 / 2} \operatorname{Sh}_{x} & =-\phi^{\prime}(0) .
\end{aligned}
$$

In the above expressions, both $\operatorname{Re}_{x}=U_{w} x / \nu$ and $\operatorname{Re}_{y}=$ $V_{w} y / \nu$ are the local Reynolds numbers.

\section{Generalized Algorithm for IPS Method}

This section explains the algorithm to generate a convergent power series for a function in general and its properties.

\section{Properties}

(1) Around $\eta=0$, the function $f(\eta)$ is expanded in a Taylor series. The infinite Taylor series is an exact representation of $f(\eta)$ for $\eta<R$, where $R$ is the radius of convergence. This Taylor series diverges when $\eta \geq R$. 
Step 1: As is customary, we expand the function $f(\eta)$ in a power series, say about $\eta=0$.

Step 2: The coefficients, $f^{(n)}(\eta)$, are then re-expressed in terms of $f(\eta)$. These creates a recursion relationship in between higherorder coefficients, $f^{(n)}(0)$, and the lower-order coefficients, $f^{(0)}(0)$, and $f^{(1)}(0)$, allowing the power series to be represented in terms of simply these two coefficients.

Step 3: The series and its derivative are then computed at $\eta=h$, where $h\left(=\eta_{\infty}-\eta_{0} / N\right)$ is substantially less than the radius of convergence of the power series.

Step 4: At $\eta=h$, a new power series expansion is performed.

Step 5: Accordingly, the higher order coefficients, $f^{(0)}(h)$ and $f^{(1)}(h)$, are re-expressed in terms of the lower-order coefficients. $f^{(0)}(h)$ and $f^{(1)}(h)$ are given the value of the previous series and its derivative determined at $\eta=h$, respectively.

Step 6: Then, around $2 h$, a new expansion is executed, using the lowest order coefficients from the preceding series, and so on. This iterative process is performed an infinite number of times. At $\eta=N h$, the final series corresponds to a convergent series.

Algorithm 1: Steps of solutions using IPS method.

(2) Truncating the Taylor series at $k_{\max }$ introduces an error of order $h^{k_{\max }+1}$. This error will be magnified $N$ times due the recursive substitutions. The total error is then estimated by

$$
\text { Error }=\left(\frac{\eta}{N}\right)^{k_{\max }+1} N
$$

For more details, the reader is referred to the reference Al-Khawaja and Al-Mdallal [38]).

\section{Numerical Method for the Solution}

This section discusses briefly the numerical algorithm used to resolve the equation (13)-(16) with the boundary conditions given by equation (17). The IPS method given by AlKhawaja and Al-Mdallal [38] and Al-Sakkaf et al. [39] will be followed. However, the shooting iteration approach for the equations (13)-(16) needs to be characterized. Hence, equations (13)-(16) are converted to IVPs of the following form:

$$
\begin{aligned}
f^{\prime \prime \prime} & =\mathbf{F}_{\mathbf{f}}\left(\eta, f, f^{\prime}, f^{\prime \prime}, g, g^{\prime}, g^{\prime \prime}\right), 0 \leq \eta \leq \infty, f(0)=0, f^{\prime}(0)=1, f^{\prime \prime}(0)=\chi, \\
g^{\prime \prime \prime} & =\mathbf{F}_{\mathbf{g}}\left(\eta, f, f^{\prime}, f^{\prime \prime}, g, g^{\prime}, g^{\prime \prime}\right), 0 \leq \eta \leq \infty, g(0)=0, g^{\prime}(0)=\alpha, g^{\prime \prime}(0)=\zeta, \\
\theta^{\prime \prime} & =\mathbf{F}_{\theta}\left(\eta, f, g, \theta, \theta^{\prime}, \phi, \phi^{\prime}\right), 0 \leq \eta \leq \infty, \theta^{\prime}(0)=-B i_{1}(1-\theta(0)), \theta(0)=\lambda, \\
\phi^{\prime \prime} & =\mathbf{F}_{\phi}\left(\eta, f, g, \theta, \theta^{\prime}, \phi, \phi^{\prime}\right), 0 \leq \eta \leq \infty, \phi^{\prime}(0)=-B i_{2}(1-\phi(0)), \phi(0)=\xi .
\end{aligned}
$$

The final values of $\chi, \zeta, \lambda$, and $\xi$ are calculated by employing an iterative procedure, so that the solutions satisfy the remaining boundary conditions, $f^{\prime}(\infty)=0$, $g^{\prime}(\infty)=0, \theta(\infty)=0$ and $\left.\phi(\infty)\right)=0$, such that

$$
\begin{aligned}
& \lim _{i \longrightarrow \infty} f^{\prime}\left(\eta_{\infty}, \chi_{i}\right)=0, \\
& \lim _{i \longrightarrow \infty} g^{\prime}\left(\eta_{\infty}, \zeta_{i}\right)=0, \\
& \lim _{i \longrightarrow \infty} \theta\left(\eta_{\infty}, \lambda_{i}\right)=0, \\
& \lim _{i \longrightarrow \infty} \phi\left(\eta_{\infty}, \xi_{i}\right)=0 .
\end{aligned}
$$

We start with appropriate initial guesses $\chi_{0}, \zeta_{0}, \lambda_{0}$, and $\xi_{0}$ to solve the IVPs (21)-(24) iteratively. The approximate solutions to $f, g, \theta, \phi$ are generated after updating the values of $\chi, \zeta, \lambda, \xi$ at each iteration step " $i$." Readers are referred to [37-39] for more details.
Denote $\mathfrak{B}=\left(\beta^{-1}+1\right)$ for simplicity. The functions $\mathbf{F}_{\mathbf{f}}, \mathbf{F}_{\mathbf{g}}, \mathbf{F}_{\theta}$, and $\mathbf{F}_{\phi}$ are presented as

$$
\begin{aligned}
& \mathbf{F}_{\mathbf{f}}=-\frac{1}{\mathfrak{B}}\left\{(f+g) f^{\prime \prime}-f^{\prime 2}-M f^{\prime}\right\}, \\
& \mathbf{F}_{\mathbf{g}}=-\frac{1}{\mathfrak{B}}\left\{(f+g) g^{\prime \prime}-g^{\prime 2}-M g^{\prime}\right\}, \\
& \mathbf{F}_{\theta}=-\left\{\operatorname{Pr}(f+g) \theta^{\prime}+\operatorname{Pr} N b \theta \prime \phi \prime+\operatorname{Pr} N t \theta^{\prime 2}+\operatorname{Pr} Q \theta e^{-m \eta}\right\}, \\
& \mathbf{F}_{\phi}=-\left\{L e(f+g) \phi^{\prime}+\frac{N t}{N b} \theta^{\prime \prime}-\operatorname{Le} \Lambda(1+\Gamma \theta)^{r} e^{(-E / 1+\Gamma \theta)}\right\} .
\end{aligned}
$$

We divide the interval $\left[0, \eta_{\infty}\right]$ into $N$ uniform subintervals $\Omega_{n}=\left[\eta_{n}, \eta_{n+1}\right]$ for $n=0,1,2,3, \ldots, N-1$ in order to solve the IVPs (21)-(24) iteratively using the IPS procedure. Let $\mathbb{Z}_{N}=\left\{\eta_{n}=n h: n=0,1,2,3, \ldots, N-1\right\}$ where $h=\eta_{\infty}-$ $\eta_{0} / N$. The exact solution of equations (21)-(24) can be shown here as a piecewise polynomial on each $\Omega_{n}$ at given $\chi=\chi_{i}, \zeta=\zeta_{i}, \lambda=\lambda_{i}$, and $\xi=\xi_{i}$ for $i \geq 1$, i.e., 


$$
\begin{aligned}
& f\left(\eta, \chi_{i}\right) \approx f_{n}(\eta)=\sum_{k=0}^{j} a_{n, k}\left(\eta-\eta_{n}\right)^{k}, \\
& g\left(\eta, \zeta_{i}\right) \approx g_{n}(\eta)=\sum_{k=0}^{j} b_{n, k}\left(\eta-\eta_{n}\right)^{k}, \\
& \theta\left(\eta, \lambda_{i}\right) \approx \theta_{n}(\eta)=\sum_{k=0}^{j-1} c_{n, k}\left(\eta-\eta_{n}\right)^{k},
\end{aligned}
$$

$$
\phi\left(\eta, \xi_{i}\right) \approx \phi_{n}(\eta)=\sum_{k=0}^{j-1} d_{n, k}\left(\eta-\eta_{n}\right)^{k} .
$$

Note that here $j$ denotes the degree of the polynomials. The functions $f, g, \theta$, and $\phi$ are assumed to be continuously differentiable on $\left[0, \eta_{\infty}\right]$ to obtain the recursion relations between the coefficients. By applying the IPS technique with $j=4$, we obtain the following recursion relations between coefficients for $n=0,1,2,3, \ldots, N-1$ (Al Sakkaf et al. [39]):

$a_{n, 0}=a_{n-1,0}+h a_{n-1,1}+h^{2} a_{n-1,2}+h^{3} a_{n-1,3}+h^{4} a_{n-1,4}$

$a_{n, 1}=a_{n-1,1}+2 h a_{n-1,2}+3 h^{2} a_{n-1,3}+4 h^{3} a_{n-1,4}$

$a_{n, 2}=a_{n-1,2}+3 h a_{n-1,3}+6 h^{2} a_{n-1,4}$

$a_{n, 3}=\frac{1}{6 \mathfrak{B}}\left\{-2 a_{n, 2}\left(a_{n, 0}+b_{n, 0}\right)+M a_{n, 1}+a_{n, 1}^{2}\right\}$,

$a_{n, 4}=-\frac{1}{24 \mathfrak{B}^{2}}\left\{-2 a_{n, 2}\left(\mathfrak{B} a_{n, 1}-\mathfrak{B} b_{n, 1}+b_{n, 0}^{2}+\mathfrak{B} M\right)+a_{n, 0}\left(-4 a_{n, 2} b_{n, 0}+M a_{n, 1}+a_{n, 1}^{2}\right)+a_{n, 1} b_{n, 0}\left(a_{n, 1}+M\right)-2 a_{n, 2} a_{n, 0}^{2}\right\}$

$b_{n, 0}=b_{n-1,0}+h b_{n-1,1}+h^{2} b_{n-1,2}+h^{3} b_{n-1,3}+h^{4} b_{n-1,4}$,

$b_{n, 1}=b_{n-1,1}+2 h b_{n-1,2}+3 h^{2} b_{n-1,3}+4 h^{3} b_{n-1,4}$

$b_{n, 2}=b_{n-1,2}+3 h b_{n-1,3}+6 h^{2} a_{n-1,4}$

$b_{n, 3}=\frac{1}{6 \mathfrak{B}}\left\{-2 b_{n, 2}\left(a_{n, 0}+b_{n, 0}\right)+M b_{n, 1}+b_{n, 1}^{2}\right\}$,

$b_{n, 4}=\frac{1}{24 \mathfrak{B}^{2}}\left\{2 \mathfrak{B} b_{n, 2}\left(-a_{n, 1}+b_{n, 1}+M\right)+2 b_{n, 0}^{2} b_{n, 2}-a_{n, 0}\left(M b_{n, 1}+b_{n, 1}^{2}-4 b_{n, 0} b_{n, 2}\right)+2 a_{n, 0}^{2} b_{n, 2}-b_{n, 0} b_{n, 1}\left(b_{n, 1}+M\right)\right\}$

$c_{n, 0}=c_{n-1,0}+h c_{n-1,1}+h^{2} c_{n-1,2}+h^{3} c_{n-1,3}+h^{4} c_{n-1,4}$,

$c_{n, 1}=c_{n-1,1}+2 h c_{n-1,2}+3 h^{2} c_{n-1,3}+4 h^{3} c_{n-1,4}$

$c_{n, 2}=-\frac{1}{2(N t \operatorname{Pr}+1)}\left\{\operatorname{Pr} e^{-h m n}\left(c_{n, 1} e^{h m n}\left(N b a_{n, 1}+b_{n, 0}+d_{n, 0}\right)+Q c_{n, 0}\right)\right\}$,

$c_{n, 3}=\frac{1}{6(N t \operatorname{Pr}+1)^{2}}\left\{\operatorname{Pr} e^{-h m n}\left(Q c_{n, 0}\left(N b \operatorname{Pr} a_{n, 1}+\operatorname{Pr} b_{n, 0}+\operatorname{Pr} d_{n, 0}+m N t \operatorname{Pr}+m\right)\right.\right.$

$-c_{n, 1}\left(-2 N b \operatorname{Pr} a_{n, 1} e^{h m n}\left(b_{n, 0}+d_{n, 0}\right)+N b^{2} \operatorname{Pr} a_{n, 1}^{2}\left(-e^{h m n}\right)\right.$

$+2 N b(N t \operatorname{Pr}+1) a_{n, 2} e^{h m n}-2 \operatorname{Pr} b_{n, 0} d_{n, 0} e^{h m n}+N t \operatorname{Pr} b_{n, 1} e^{h m n}-\operatorname{Pr} b_{n, 0}^{2} e^{h m n}+b_{n, 1} e^{h m n}$

$\left.\left.\left.+N t \operatorname{Pr} d_{n, 1} e^{h m n}-\operatorname{Pr} d_{n, 0}^{2} e^{h m n}+d_{n, 1} e^{h m n}+N t \operatorname{PrQ}+Q\right)\right)\right\}$,

$d_{n, 0}=d_{n-1,0}+h d_{n-1,1}+h^{2} d_{n-1,2}+h^{3} d_{n-1,3}+h^{4} d_{n-1,4}$,

$d_{n, 1}=d_{n-1,1}+2 h d_{n-1,2}+3 h^{2} d_{n-1,3}+4 h^{3} d_{n-1,4}$, 


$$
\begin{aligned}
d_{n, 2}= & \frac{1}{2 N t}\left\{N b\left(\operatorname{Le}\left(\Gamma e^{-E / \Gamma d_{n, 0}+1}\left(\Gamma d_{n, 0}+1\right)^{r}-a_{n, 1}\left(b_{n, 0}+c_{n, 0}\right)\right)-2 a_{n, 2}\right)\right\} \\
d_{n, 3}= & \frac{1}{6 N t}\left\{N b \left(-\frac{M a_{n, 1}+a_{n, 1}^{2}-2 a_{n, 2}\left(a_{n, 0}+b_{n, 0}\right)}{\mathfrak{B}}-2 \operatorname{Le} a_{n, 2} b_{n, 0}-\operatorname{Le} a_{n, 1} b_{n, 1}-\operatorname{Le}\left(2 a_{n, 2} c_{n, 0}+a_{n, 1} c_{n, 1}\right)\right.\right. \\
& \left.\left.+\Gamma^{2} \operatorname{Led}_{n, 1} e^{-E / \Gamma d_{n, 0}+1}\left(\Gamma d_{n, 0}+1\right)^{r-2}\left(\Gamma r d_{n, 0}+E+r\right)\right)\right\} .
\end{aligned}
$$

The process continues until the boundary conditions, equations (25)-(28), are satisfied, i.e., $\left|f^{\prime}\left(\eta_{\infty}, \chi_{i}\right)\right|<\varepsilon$, $\left|g^{\prime}\left(\eta_{\infty}, \zeta_{i}\right)\right|<\varepsilon,\left|\theta\left(\eta_{\infty}, \lambda_{i}\right)\right|<\varepsilon$, and $\left|\phi\left(\eta_{\infty}, \xi_{i}\right)\right|<\varepsilon$. Here $\varepsilon$ is the tolerance fixed to $10^{-10}$, and the optimal choice for $\eta_{\infty}$ is discussed in Section 7.

\section{Validation}

In the present section, we intend to assess the performance and efficiency of the present numerical scheme for solving (21)-(24). We will concentrate on equation (21), which can be written as

$$
\left(\beta^{-1}+1\right) f^{\prime \prime \prime}+(f+g) f^{\prime \prime}-f^{\prime 2}-M f^{\prime}=0,
$$

with the conditions

$$
f(0)=0, f^{\prime}(0)=1, f^{\prime \prime}(0)=\chi,
$$

where the value $\chi$ is modified using the shooting technique in order to satisfy $f^{\prime}(\infty)=0$.

We discuss the relevance of choosing the numerical parameter, $\eta_{\infty}$, in order achieve accurate numerical data. Table 2 presents the values of $\chi$ as a function of $\eta$ for distinct values of $\eta_{\infty}$ in order to obtain an optimal value of $\eta_{\infty}$. As mentioned earlier, the values of the parameter $\chi$ are updated at every iteration until it becomes sufficiently close to $f^{\prime}(\infty)=0$. Table 2 shows that the values of $\chi$ become nearly constant after few iterations as $\eta_{\infty}>7$. Therefore, $\eta_{\infty}=15$ is the optimal value. However, the range of values up to $\eta_{\infty}=7$ is considered for plotting the figures.

The associated residuals to (21)-(24) are, respectively, given by

$$
\begin{aligned}
& \operatorname{Res}_{f}(\eta)=\left(\beta^{-1}+1\right) f^{\prime \prime \prime}+(f+g) f^{\prime \prime}-f^{\prime 2}-M f^{\prime}, \\
& \operatorname{Res}_{g}(\eta)=\left(\beta^{-1}+1\right) g^{\prime \prime \prime}+(f+g) g^{\prime \prime}-g^{\prime 2}-M g^{\prime} \\
& \operatorname{Res}_{\theta}(\eta)=\theta^{\prime \prime}+\operatorname{Pr}(f+g) \theta^{\prime}+\operatorname{Pr} N b \theta^{\prime} \phi^{\prime}+\operatorname{Pr} N t \theta^{\prime 2}+\operatorname{PrQ} \theta e^{-m \eta}, \\
& \operatorname{Res}_{\phi}(\eta)=\phi^{\prime \prime}+\operatorname{Le}(f+g) \phi^{\prime}+\frac{N t}{N b} \theta^{\prime \prime}-L e \Lambda(1+\Gamma \theta)^{r} e^{(-E / 1+\Gamma \theta)}
\end{aligned}
$$

In Table 3, we present the residual error values for $\operatorname{Res}_{f}(\eta)$ in equation (54) corresponding to each $\eta_{\infty}$ using the IPS method. The findings demonstrate an accuracy of order $10^{-10}$.

To guarantee the convergence of the solution, we present Tables 4 and 5 , which compare the numerical results obtained for $f^{\prime}(\eta)$ and $g^{\prime}(\eta)$ using the IPS method with the approximate solution derived using Runge-Kutta method of fourth order for various values of $\eta$.

To validate our numerical solution, the estimated numerical results for $f^{\prime \prime}(0)$ and $g^{\prime \prime}(0)$ are compared with the available literature. Table 6 compares $f^{\prime \prime}(0)$ and $g^{\prime \prime}(0)$ values obtained in the present research with those of Umar et al. [19], Freidoonimehr and Rahimi [12], and Wang [11]. It is clear that the results are in very good agreement with an accuracy of $10^{-5}$.

\section{Results and Discussion}

The results obtained for equations (13)-(16) by transforming into IVPs given by equations (21)-(24) and using the IPS method combined with the shooting approach are discussed in this section. Now onwards, $f^{\prime}(\eta), g^{\prime}(\eta), \theta(\eta)$, and $\phi(\eta)$ have been used to mention the $x$ component of velocity, $y$ component of the velocity, temperature, and concentration profiles, respectively. Similarly, we mention momentum boundary layer as MBL, thermal boundary layer as TBL, and concentration boundary layer as CBL, respectively. The parameter are values are varied between the following ranges: $0.5 \leq M \leq 2.0, \quad 0.6 \leq \alpha \leq 0.9, \quad 2.0 \leq Q \leq 5.0, \quad 0.1 \leq E \leq 0.25$, $0.1 \leq M \leq 0.25,1.0 \leq B i_{1} \leq 2.5,0.5 \leq B i_{2} \leq 0.8,2.0 \leq N b \leq 5.0$, $0.2 \leq N t \leq 0.8, \quad 0.5 \leq \Gamma \leq 2.0, \quad 0.6 \leq L e \leq 0.75, \quad 3.0 \leq \operatorname{Pr} \leq 4.5$, and $0.1 \leq \Lambda \leq 0.25$. The value of $\beta$ is fixed to 0.5 and $m$ and $n$ to 1 throughout this study.

Table 7 presents the calculated values of the local skin friction coefficients in the $x$-and $y$-directions. Following the effects of the Casson parameter $(\beta), \operatorname{Re}_{x}^{1 / 2} C_{f x}$ and $\alpha^{3 / 2}, \operatorname{Re}_{y}^{1 / 2} C_{f y}$ are enhanced, while it is decreased following the effect of magnetic parameter $(M)$ in both directions. Interestingly, for the case of $\alpha$, the friction coefficient, 
TABLE 2: Values of parameter $\chi$ corresponding to $\eta_{\infty}$, calculated by using shooting method with $M=1.0, \beta=0.5, \alpha=0.3$, and absolute error in the subsequent values of $\eta_{\infty}$.

\begin{tabular}{lcc}
\hline$\eta_{\infty}$ & $\chi$ & Absolute Error \\
\hline 7 & -0.92126708636310317 & - \\
8 & -0.92113408041491218 & $1.33 \times 10^{-4}$ \\
9 & -0.92109189943454539 & $4.22 \times 10^{-5}$ \\
10 & -0.92107847137819310 & $1.34 \times 10^{-5}$ \\
11 & -0.92107419566890625 & $4.28 \times 10^{-6}$ \\
12 & -0.92107282577059646 & $1.37 \times 10^{-6}$ \\
13 & -0.92107238794947266 & $4.38 \times 10^{-7}$ \\
14 & -0.92107224800468002 & $1.40 \times 10^{-7}$ \\
15 & -0.92107220327054939 & $4.47 \times 10^{-8}$ \\
\hline
\end{tabular}

TABLE 3: Residual error $\left(\operatorname{Res}_{f}(\eta)\right)$ corresponding to $\eta_{\infty}$, calculated by using IPS method with $M=1.0, \beta=0.5$, and $\alpha=0.3$.

\begin{tabular}{lc}
\hline$\eta_{\infty}$ & Error \\
\hline 7 & 0.0000130877 \\
8 & $2.50503 \times 10^{-7}$ \\
9 & $-2.01555 \times 10^{-7}$ \\
10 & $2.81436 \times 10^{-6}$ \\
11 & $-1.68648 \times 10^{-7}$ \\
12 & $-6.91890 \times 10^{-7}$ \\
13 & $-3.96241 \times 10^{-8}$ \\
14 & $-1.14731 \times 10^{-7}$ \\
15 & $-8.83440 \times 10^{-10}$ \\
\hline
\end{tabular}

TABLE 4: Estimations of error for $f^{\prime}(\eta)$ by setting $M=0.5, \alpha=0.5$, and $\beta=0.5$.

\begin{tabular}{lccc}
\hline$\eta$ & $f_{\mathrm{IPS}}^{\prime}(\eta)$ & $f_{\mathrm{RK} 4}^{\prime}(\eta)$ & Error $\left(f_{\mathrm{IPS}}^{\prime}(\eta)-f_{\mathrm{RK} 4}^{\prime}(\eta)\right)$ \\
\hline 1 & 1.000000000000 & 1.000000000000 & 0.00 \\
2 & 0.460525220817 & 0.460525181476 & $3.9341 \times 10^{-8}$ \\
3 & 0.204915558646 & 0.204915533345 & $2.53006 \times 10^{-8}$ \\
4 & 0.089670147015 & 0.089670097323 & $4.96921 \times 10^{-8}$ \\
5 & 0.038942267210 & 0.038942231873 & $3.53371 \times 10^{-8}$ \\
6 & 0.016855052694 & 0.016855116531 & $-6.38367 \times 10^{-8}$ \\
7 & 0.007284282287 & 0.007284365925 & $-8.36375 \times 10^{-7}$ \\
8 & 0.003145699764 & 0.003145804840 & $-1.05076 \times 10^{-7}$ \\
9 & 0.001357621734 & 0.001357770701 & $-1.48967 \times 10^{-7}$ \\
10 & 0.000585300711 & 0.000585480549 & $-1.79839 \times 10^{-7}$ \\
\hline
\end{tabular}

TABLE 5: Estimations of error for $g^{\prime}(\eta)$ by setting $M=0.5, \alpha=0.5$, and $\beta=0.5$.

\begin{tabular}{lccc}
\hline$\eta$ & $g_{\mathrm{IPS}}^{\prime}(\eta)$ & $g_{\mathrm{RK} 4}^{\prime}(\eta)$ & Error $\left(g_{\mathrm{IPS}}^{\prime}(\eta)-g_{\mathrm{RK} 4}^{\prime}(\eta)\right)$ \\
\hline 1 & 0.500000000000 & 0.500000000000 & 0.00 \\
2 & 0.243218922052 & 0.243218869936 & $5.21155 \times 10^{-8}$ \\
3 & 0.110964835767 & 0.110964849780 & $-1.40132 \times 10^{-8}$ \\
4 & 0.049098390961 & 0.049098373377 & $1.75844 \times 10^{-8}$ \\
5 & 0.021425892132 & 0.021425880937 & $1.11958 \times 10^{-8}$ \\
6 & 0.009293015758 & 0.009293079577 & $-6.38193 \times 10^{-8}$ \\
7 & 0.004019793239 & 0.004019881570 & $-8.8331 \times 10^{-8}$ \\
8 & 0.001736581704 & 0.001736693904 & $-1.122 \times 10^{-7}$ \\
9 & 0.000749555748 & 0.000749706482 & $-1.50734 \times 10^{-7}$ \\
10 & 0.000323117550 & 0.000323302343 & $-1.84793 \times 10^{-7}$ \\
\hline
\end{tabular}

$\operatorname{Re}_{x}^{1 / 2} C_{f x}$, increases, and $\alpha^{3 / 2} \operatorname{Re}_{y}^{1 / 2} C_{f y}$ decreases according to the directions. Table 8 lists the numerical values for the local Nusselt number and local Sherwood number for different values of the relevant physical parameters. From this table, it is clear that the heat transfer rate increases with the thermal Biot number $\left(B i_{1}\right)$, exponential heat source parameter $(Q)$, and Lewis number $(L e)$. Likewise, the local Sherwood number shows an enhancement with the concentration Biot number $\left(B i_{2}\right)$, Lewis number, $(L e)$, and reaction rate constant $(\Lambda)$. It is noteworthy to mention that the local Nusselt and Sherwood numbers reduce following the effects of Arrhenius activation energy.

Figures 2-5 present the behavior of the velocity profiles in $x$ and $y$ directions $\left(f^{\prime}(\eta)\right.$ and $g^{\prime}(\eta)$, respectively), temperature $(\theta(\eta))$, and concentration $(\phi(\eta))$ profiles with respect to $\eta$ for the variations in the magnetic parameter $(M)$ and stretching ratio parameter $(\alpha)$. It is observed that $f^{\prime}(\eta)$ and $g^{\prime}(\eta)$ show a gradual decrease in the flow domain with an increase in the values of $M$ from 0.5 to 2 . A charged molecule moving across a magnetic field is acted upon by a force that is perpendicular to the direction applied field and to the direction in which the particle is moving. The interactions between the applied magnetic field and the magnetic field generated by the moving particle lead to development of a force, termed as the Lorentz force. This force acts as a drag-like force, opposite to the direction of fluid motion. Therefore, an increase in the strength of the magnetic field applied in the direction perpendicular to the fluid flow tends to reduce the fluid velocities along both $x$-and $y$-directions, as depicted in Figures 2 and 3. Similar observations are reported by Nadeem et al. [26]. Lorentz forces tend to increase the heat transmission in the fluid domain by considerably reducing the flow velocities. Hence, $\theta(\eta)$ enhances significantly with $M$ as shown in Figure 4. Even though the temperature distribution under the influence of $M$ is considerably high, the concentration profile of the fluid reduces considerably with an increase in $M$ as depicted in Figure 5.

Figures 2-5 also demonstrate the effect of $\alpha$ on the parameters $f^{\prime}(\eta), g^{\prime}(\eta), \theta(\eta)$, and $\phi(\eta)$. A gradual increase in $\alpha$ from 0.6 to 0.9 leads to an enhancement in the transverse velocity $g^{\prime}(\eta)$, whereas $f^{\prime}(\eta)$ diminishes. $\alpha(=b / a)$ is a quantitative relation between the stretching velocities in $x$ and $y$ directions, represented as $U_{w}=a x$ and $V_{w}=b y$. On increasing $\alpha$, the sheet stretching force significantly increases in the transverse direction. This is attributed to the relation of direct proportionality between $\alpha$ and the transverse velocity $\left(g^{\prime}(\eta)\right)$. On the other hand, the axial velocity field $\left(f^{\prime}(\eta)\right)$, which is inversely proportional to $\alpha$, decreases with an increase in $\alpha$ (see Figures 2 and 3). The enhanced values of $\alpha$ seem not to influence the TBL, and $\theta(\eta)$ continues to decline. This is due to the fact that the cooler fluid from the ambient region progresses towards the sheet relatively faster due to the stretching of the sheet. As a result the temperature gradient near the sheet is increased and thus the TBL grows thinner. This leads to decreased temperature profile (see Figure 4). In addition, stretching of the sheet leads to a decrease in the values of $\phi(\eta)$. The CBL thickness decreases with an increase in the values of $\alpha$ in Figure 5. 
TABLE 6: Comparison of the present numerical results for $\alpha, f^{\prime \prime}(0)$, and $g^{\prime \prime}(0)$ with Umar et al. [19], Freidoonimehr and Rahimi [12], and Wang [11].

\begin{tabular}{ccccccc}
\hline & $\alpha$ & Umar et al. [19] & Freidoonimehr and Rahimi [12] & Wang [11] & Present Result & Maximum Error \\
\hline & 0 & -1.0000 & -1.0000 & -1.0000 & -1.0000000 & - \\
$f^{\prime \prime}(0)$ & 0.25 & -1.0490 & -1.04881 & -1.0488 & -1.0488110 & $-1.1 \times 10^{-5}$ \\
& 0.5 & -1.0932 & -1.09309 & -1.0930 & -1.0930950 & $-9.5 \times 10^{-5}$ \\
& 0.75 & -1.1345 & -1.13450 & -1.1344 & -1.1344857 & $-8.57 \times 10^{-5}$ \\
& 1.0 & -1.1737 & -1.17372 & -1.1737 & -1.1737207 & $-2.07 \times 10^{-5}$ \\
\hline & 0 & 0.00000 & 0.00000 & 0.0000 & 0.0000000 & - \\
$g^{\prime \prime}(0)$ & 0.25 & -0.19457 & -0.19457 & -0.1945 & -0.1945638 & $-1.38 \times 10^{-3}$ \\
& 0.5 & -0.46532 & -0.46520 & -0.4652 & -0.4652048 & $-4.8 \times 10^{-6}$ \\
& 0.75 & -0.79470 & -0.79462 & -0.7946 & -0.7946182 & $-1.8 \times 10^{-5}$ \\
& 1.0 & -1.17372 & -1.17372 & -1.1737 & -1.1737207 & $-2.07 \times 10^{-5}$ \\
\hline
\end{tabular}

TABLe 7: Values of $\operatorname{Re}_{x}^{1 / 2} C_{f x}$ and $\alpha^{3 / 2} \operatorname{Re}_{y}^{1 / 2} C_{f y}$ corresponding to $M, \alpha$, and $\beta$.

\begin{tabular}{ccccc}
\hline$M$ & $\alpha$ & $\beta$ & $\operatorname{Re}_{x}^{1 / 2} C_{f x}$ & $\alpha^{3 / 2} \mathrm{Re}_{y}^{1 / 2} C_{f y}$ \\
\hline 0.0 & & & -0.91263354 & -0.10486921 \\
0.3 & & -1.14686424 & -0.19773170 \\
0.6 & & -1.36502137 & -0.28057038 \\
& 0.0 & & -1.67539238 & -0.00000000 \\
& 0.25 & & -1.64024018 & -0.30972771 \\
& 0.5 & 0.5 & -1.60811195 & -0.67453871 \\
& & 1.0 & -1.63359047 & -0.37845134 \\
& & 3.0 & -1.28109625 & -0.29421997 \\
\hline
\end{tabular}

TABLE 8: Values of $\mathrm{Re}_{x}^{-1 / 2} \mathrm{Nus}_{x}$ and $\mathrm{Re}_{x}^{-1 / 2} \mathrm{Sh}_{x}$ corresponding to $M, B i_{1}, B i_{2}, N b, N t, L e, E, \Lambda, Q$, and $\beta$.

\begin{tabular}{|c|c|c|c|c|c|c|c|c|c|c|c|}
\hline$M$ & $B i_{1}$ & $B i_{2}$ & $\mathrm{Nb}$ & $N t$ & Le & $E$ & $\Lambda$ & $Q$ & $\beta$ & $\mathrm{Re}_{x}^{-1 / 2} \mathrm{Nus}_{x}$ & $\mathrm{Re}_{x}^{-1 / 2} \mathrm{Sh}_{x}$ \\
\hline 0.0 & & & & & & & & & & 0.73049067 & 0.16449552 \\
\hline 0.3 & & & & & & & & & & 0.72902988 & 0.17600451 \\
\hline \multirow[t]{28}{*}{0.6} & & & & & & & & & & 0.72753576 & 0.18873343 \\
\hline & 0.1 & & & & & & & & & 0.09741008 & 0.23319547 \\
\hline & 0.2 & & & & & & & & & 0.18945977 & 0.22896889 \\
\hline & 0.3 & & & & & & & & & 0.27593479 & 0.22506826 \\
\hline & & 0.1 & & & & & & & & 0.72556403 & 0.20629971 \\
\hline & & 0.2 & & & & & & & & 0.71283726 & 0.39898561 \\
\hline & & 0.3 & & & & & & & & 0.70031755 & 0.57950017 \\
\hline & & & 0.1 & & & & & & & 0.72941262 & 0.07371255 \\
\hline & & & 0.2 & & & & & & & 0.72846414 & 0.15657457 \\
\hline & & & 0.3 & & & & & & & 0.72750619 & 0.18419747 \\
\hline & & & & 0.1 & & & & & & 0.78918148 & 0.23318320 \\
\hline & & & & 0.2 & & & & & & 0.77992473 & 0.22807811 \\
\hline & & & & 0.3 & & & & & & 0.77015236 & 0.22320023 \\
\hline & & & & & 0.5 & & & & & 0.72556403 & 0.20629971 \\
\hline & & & & & 1.0 & & & & & 0.73741327 & 0.22744454 \\
\hline & & & & & 1.5 & & & & & 0.74486116 & 0.23559864 \\
\hline & & & & & & 0.1 & & & & 0.72563138 & 0.23770808 \\
\hline & & & & & & 0.2 & & & & 0.72560205 & 0.22122394 \\
\hline & & & & & & 0.3 & & & & 0.72556403 & 0.20629971 \\
\hline & & & & & & & 0.1 & & & 0.72437632 & 0.09197616 \\
\hline & & & & & & & 0.2 & & & 0.72469919 & 0.12056004 \\
\hline & & & & & & & 0.3 & & & 0.72500447 & 0.14914185 \\
\hline & & & & & & & & 0.1 & & 0.74194053 & 0.20491889 \\
\hline & & & & & & & & 0.2 & & 0.73812455 & 0.20524079 \\
\hline & & & & & & & & 0.3 & & 0.73413256 & 0.20557744 \\
\hline & & & & & & & & & 0.5 & 0.72556403 & 0.20629971 \\
\hline & & & & & & & & & 1.0 & 0.72425929 & 0.21507536 \\
\hline & & & & & & & & & 3.0 & 0.72287366 & 0.22407199 \\
\hline
\end{tabular}




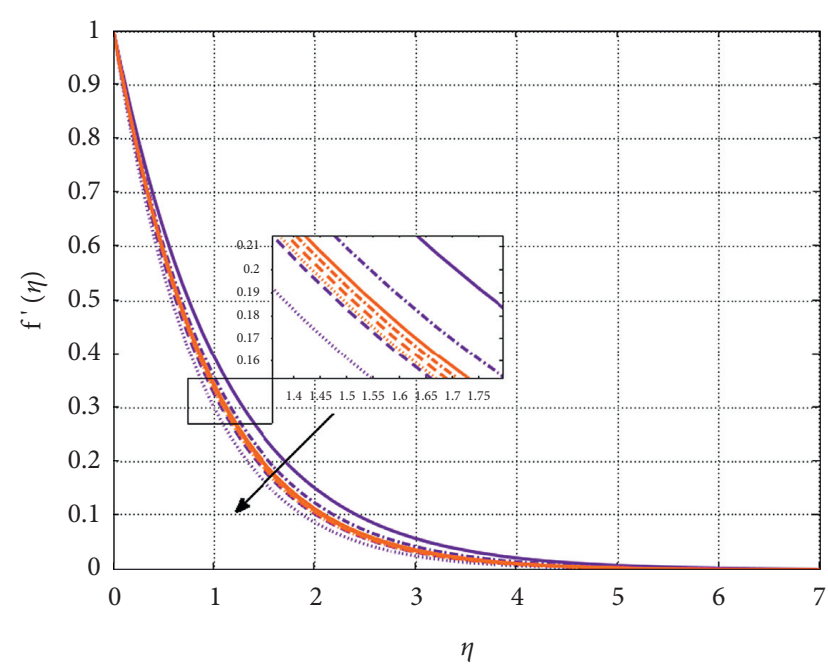

$$
\begin{array}{rlr}
-\mathrm{M} & =1.0 & -\alpha=0.6 \\
\ldots-\mathrm{M} & =1.5 & -\alpha=0.7 \\
--\mathrm{M}=2.0 & ---\alpha=0.8 \\
\ldots \ldots \mathrm{M}=2.5 & \ldots \ldots \alpha=0.9
\end{array}
$$

FIgURE 2: Behavior of $f^{\prime}(\eta)$ with respect to $\eta$ for $1 \leq M \leq 2.5$ and $0.6 \leq \alpha \leq 0.9$.

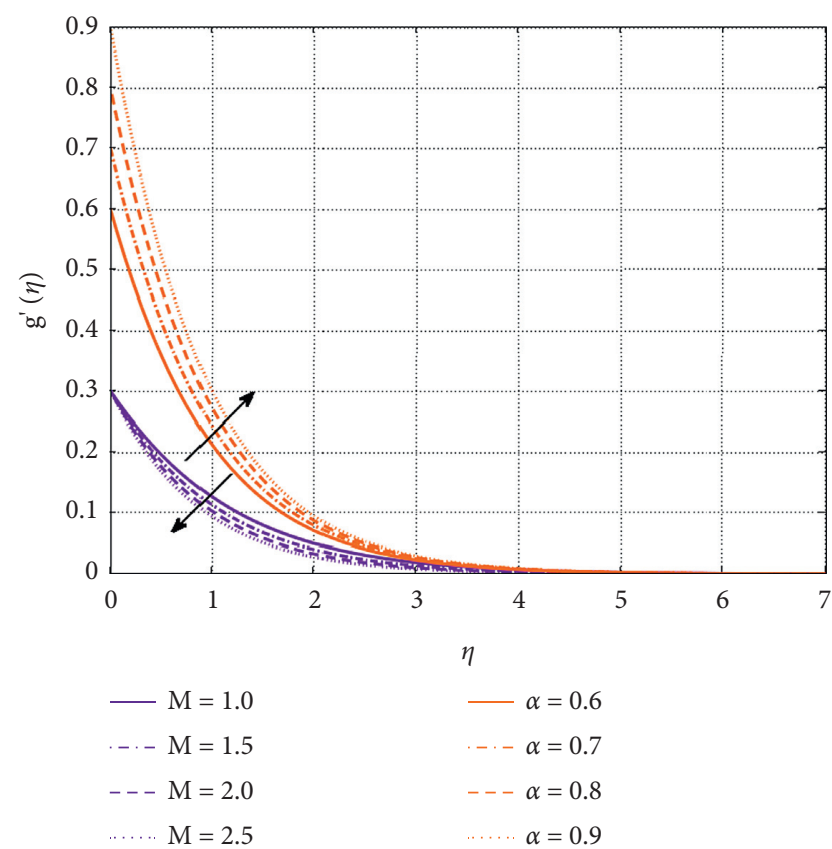

Figure 3: Behavior of $g^{\prime}(\eta)$ with respect to $\eta$ for $1 \leq M \leq 2.5$ and $0.6 \leq \alpha \leq 0.9$.

Figures 6 and 7 present the impact of the exponential heat source parameter $(Q)$ and the Brownian motion parameter $(N b)$ on the parameters $\theta(\eta)$ and $\phi(\eta)$, respectively. The exponential heat source provides heat to the fluid, leading to a strong thermal environment, which justifies the behavior of $Q$ with $\theta(\eta)$. Similarly, $N b$ displays an opposite

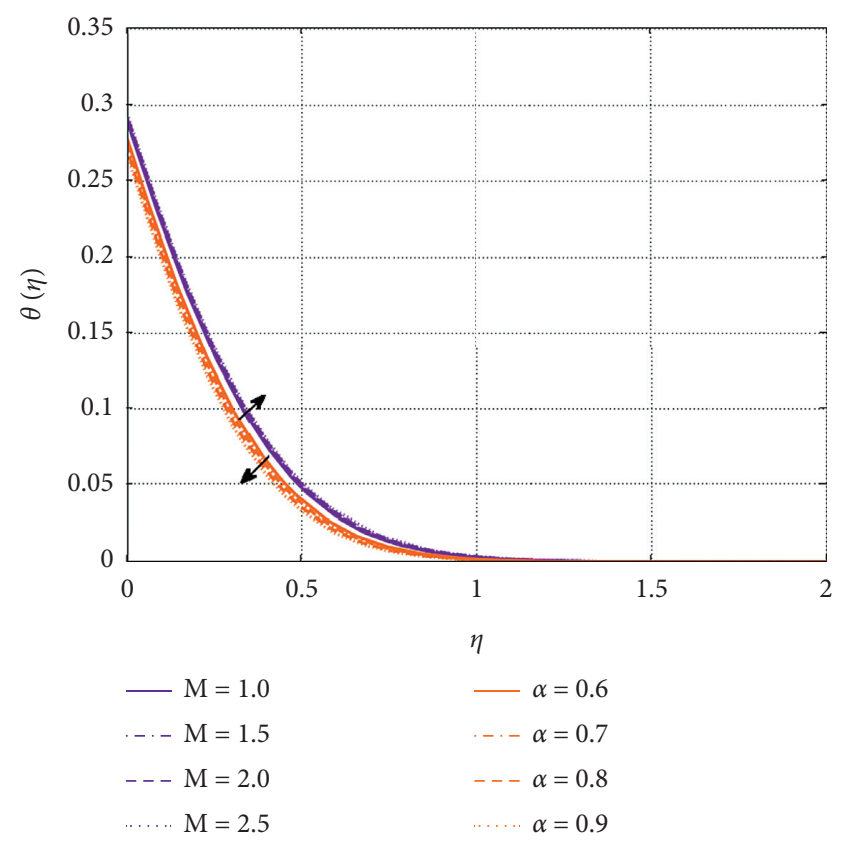

FIgURE 4: Behavior of $\theta(\eta)$ with respect to $\eta$ for $1 \leq M \leq 2.5$ and $0.6 \leq \alpha \leq 0.9$.

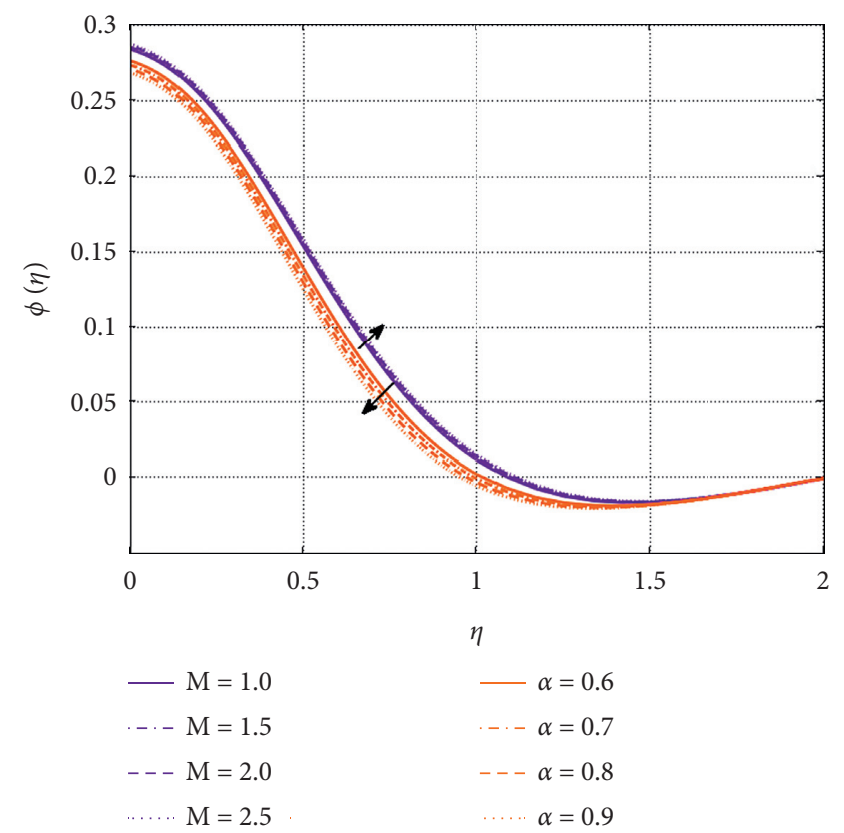

FIgURE 5: Behavior of $\phi(\eta)$ with respect to $\eta$ for $1 \leq M \leq 2.5$ and $0.6 \leq \alpha \leq 0.9$.

behavior with $\theta(\eta)$. The unpredictable migration of fluid molecules increases with increasing $N b$. This migration can be attributed to a reduction in the TBL thickness, which eventually leads to a decrease in the temperature in the whole domain.

In Figure 7, the curves of the concentration profile can be analyzed with values of $Q$ ranging from 2.0 to 5.0. Initially, 


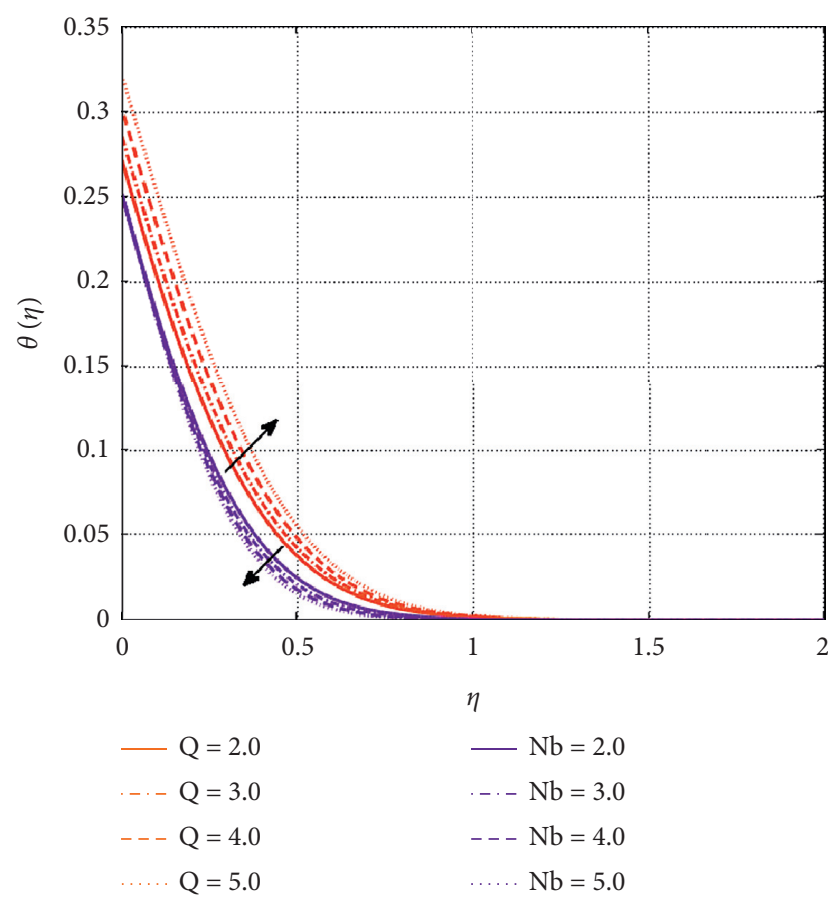

Figure 6: Behavior of $\theta(\eta)$ with respect to $\eta$ for $2 \leq Q \leq 5.0$ and $2.0 \leq N b \leq 5.0$.

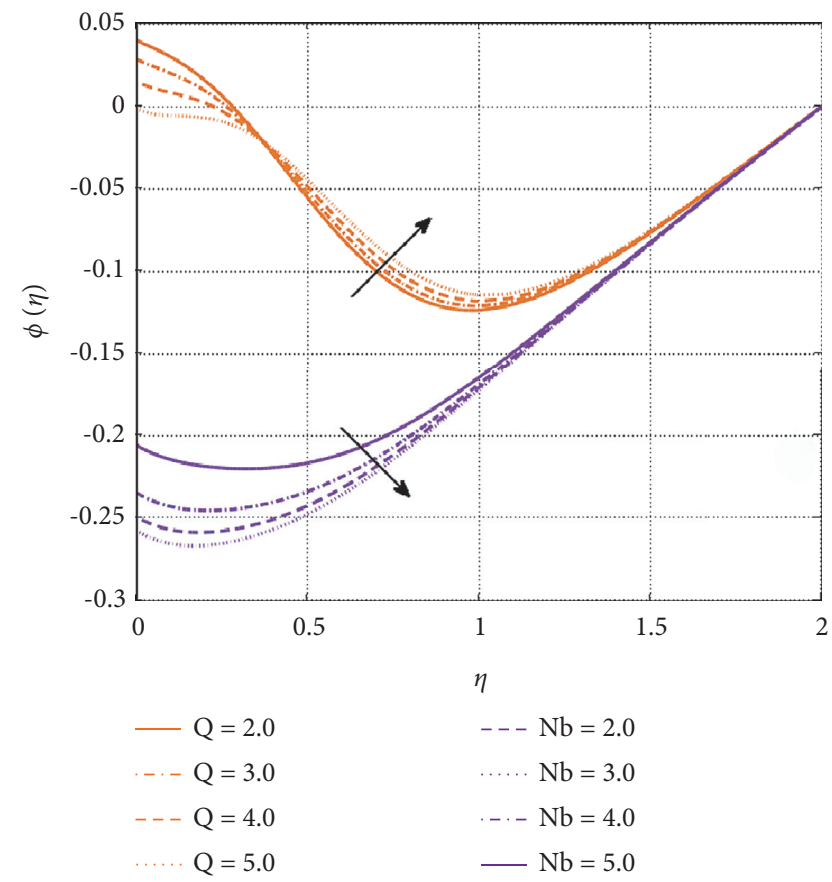

FIgURE 7: Behavior of $\phi(\eta)$ with respect to $\eta$ for $2 \leq Q \leq 5.0$ and $2.0 \leq N b \leq 5.0$.

the concentration decreases in the region close to the sheet surface $(0 \leq \eta \leq 0.4)$, and as $\eta$ approaches the free stream region, the concentration increases. This is due to the fact that the particles in the liquids remain very close to each other near the surface that tend to increase the concentration gradient. This increment tends to a decrease in the TBL thickness and it further affects the temperature profile in the vicinity of the sheet surface. But, as the fluid particles move at a considerable distance from the sheet surface, concentration gradient effects are less perceived and therefore the TBL thickness is larger here. Hence, the concentration profiles far away from the sheet show an increase. We also observe that an increase in the Brownian motion parameter $(\mathrm{Nb})$ tends to reduce the concentration profile. Mechanically, $\mathrm{Nb}$ tends to induce a continual acceleration between the fluid molecules. Such an acceleration boosted particles move faster as they acquire kinetic energy, which leads to higher collision rates and an increased diffusion rate. Thus, the greater the migration of particles to regions of lower concentration, the lower the values of $\phi(\eta)$.

Figures 8 and 9 present the behavior of $\theta(\eta)$ and $\phi(\eta)$ against the Prandtl number (Pr) and the thermophoresis parameter $(N t), \mathrm{Pr}$, which is represented as the ratio of energy diffusiveness to warm diffusiveness, and helps to reduce $\theta(\eta)$ and $\phi(\eta)$. The general thickening of MBL, TBL, and CBL in heat transfer problems is well known to be controlled by $\operatorname{Pr}$ (Shehzad et al. [27]). If Pr is small (smaller than 1), the MBL is considerably less than those of the TBL and CBL. Thus, increasing the values of $\mathrm{Pr}$ from 3.0 to 4.5 may decrease the thicknesses of TBL and CBL. This leads to a reduction with $\theta(\eta)$ and $\phi(\eta)$. Similar observations have been documented by Shehzad et al. [27].

Figures 8 and 9 depict the variations in the thermal and concentration profiles with respect to $\mathrm{Nt}$. If a temperature gradient exists in a liquid, suspended particles tend to move from a region of high temperature to a region of low temperature. The force responsible for this behaviour is termed as thermophoretic force. Particles colliding with the particles lying in region of high temperature have higher velocities than those colliding with the particles lying in the region of lower temperature. This leads to a net force towards the low temperature regions. Thus, more fluid gets heated that leads to a rise in the temperature, as observed in Figure 8. However, in Figure 9, an increase in $\phi(\eta)$ has been observed. This is attributed to the fact that the thermophoresis present in the domain tends to agitate the particles. The random oscillations do not possess a particular direction. Hence, the particles tend to spread equally throughout the domain over a period of time, from regions of high concentration to regions of lower concentration. This phenomenon is also called diffusion. Therefore, diffusion tends to improve the concentration boundary layer and it leads to an increase in $\phi(\eta)$, as depicted in Figure 9.

Figure 10 shows the dynamics of the Arrhenius activation energy parameter $(E)$ and the reaction rate constant $(\Lambda)$ with $\phi(\eta)$. The expression, $E=E_{a} / k_{1} T_{\infty}$, describes the connection of temperature with the speed of a chemical reaction. Here, $E_{a}$ denotes the activation energy for the reaction and $k_{1}$ denotes the reaction rate constant. As the activation energy increases, the generative chemical reaction is initiated, which leads to an enhanced concentration profile. Meanwhile, an increase in $\Lambda$ results in the decrease of $\phi(\eta)$ to ensure an enormous concentration gradient on the wall, which lowers the thickness of CBL. 


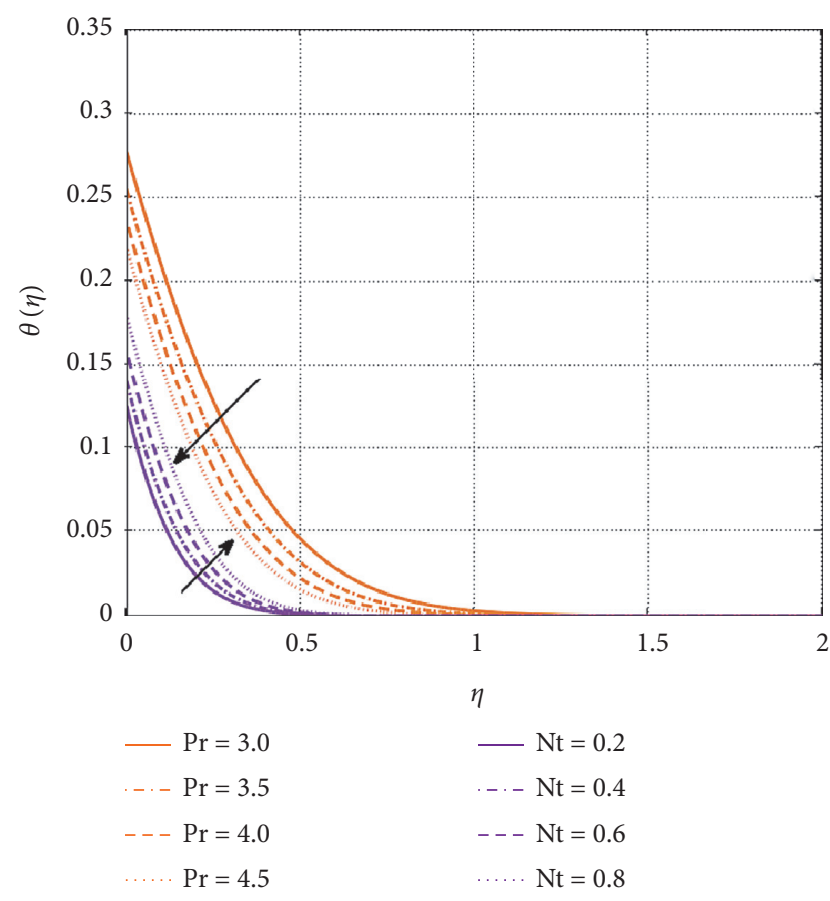

Figure 8: Behavior of $\theta(\eta)$ with respect to $\eta$ for $3.0 \leq \operatorname{Pr} \leq 4.5$ and $0.2 \leq N t \leq 0.8$.

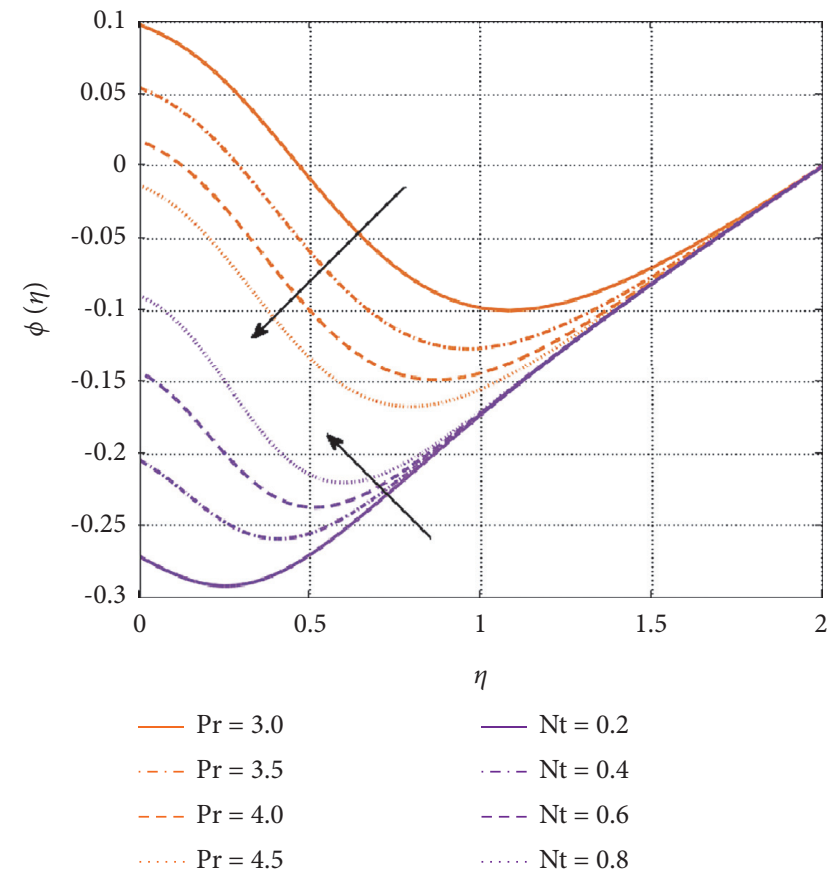

Figure 9: Behavior of $\phi(\eta)$ with respect to $\eta$ for $3.0 \leq \operatorname{Pr} \leq 4.5$ and $0.2 \leq N t \leq 0.8$.

Figures 11 and 12 present the impacts of $B i_{1}$ and $B i_{2}$ on $\theta(\eta)$ and $\phi(\eta)$, respectively. Here, $B i_{1}$ and $B i_{2}$ denote the thermal and concentration Biot numbers, respectively. Similarly, the fixed temperature of the wall $\theta(0)=1$ and the concentration $\phi(0)=1$ are achieved using the values of $B i_{1}$ and $B i_{2}$. An increment in the values of $B i_{1}$ and $B i_{2}$ leads to an

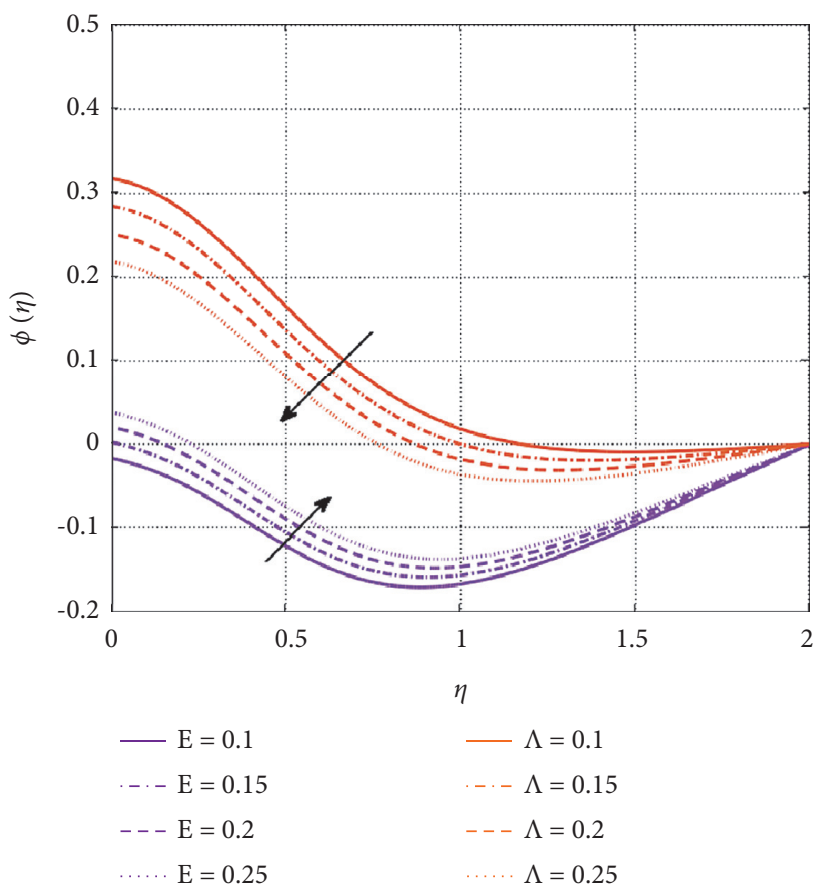

FIgURE 10: Behavior of $\phi(\eta)$ with respect to $\eta$ for $0.1 \leq E \leq 4.5$ and $0.1 \leq \Lambda \leq 0.25$.

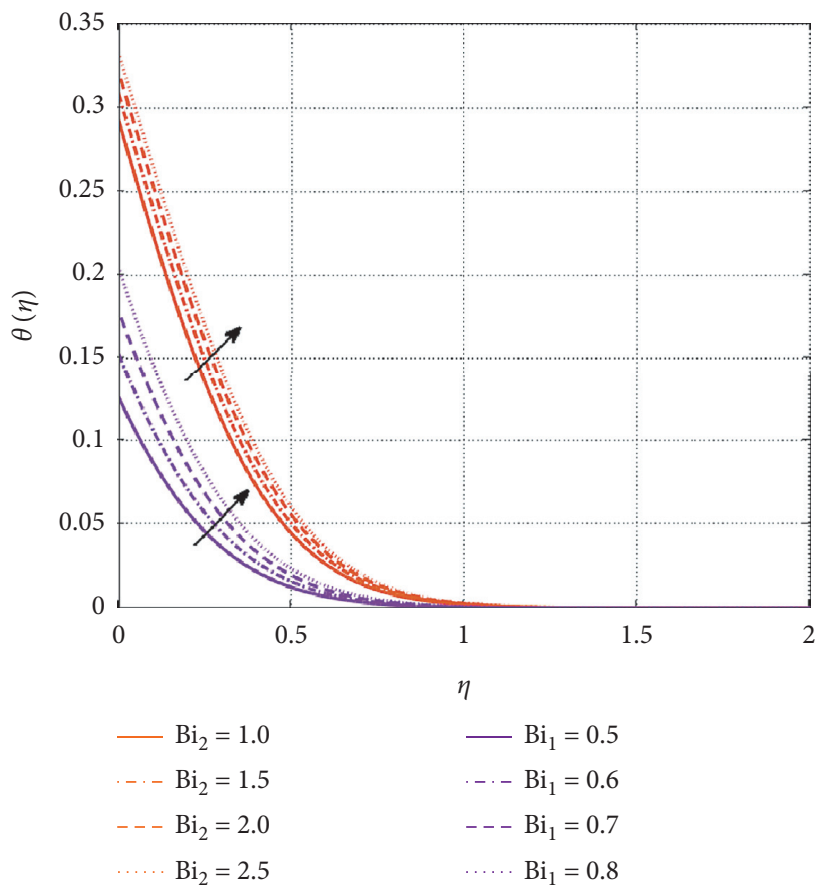

FIGURE 11: Behavior of $\theta(\eta)$ with respect to $\eta$ for $1.0 \leq B i_{1} \leq 2.5$ and $0.5 \leq B i_{2} \leq 0.8$.

increase in the values of heat and mass transfer coefficients. This increase in the values of these coefficients enhances the temperature and the concentration profiles. The variations in $\phi(\eta)$ against the nondimensional fluid parameters $(\Gamma)$ and Lewis number $(L e)$ are depicted in Figure 13. This figure 


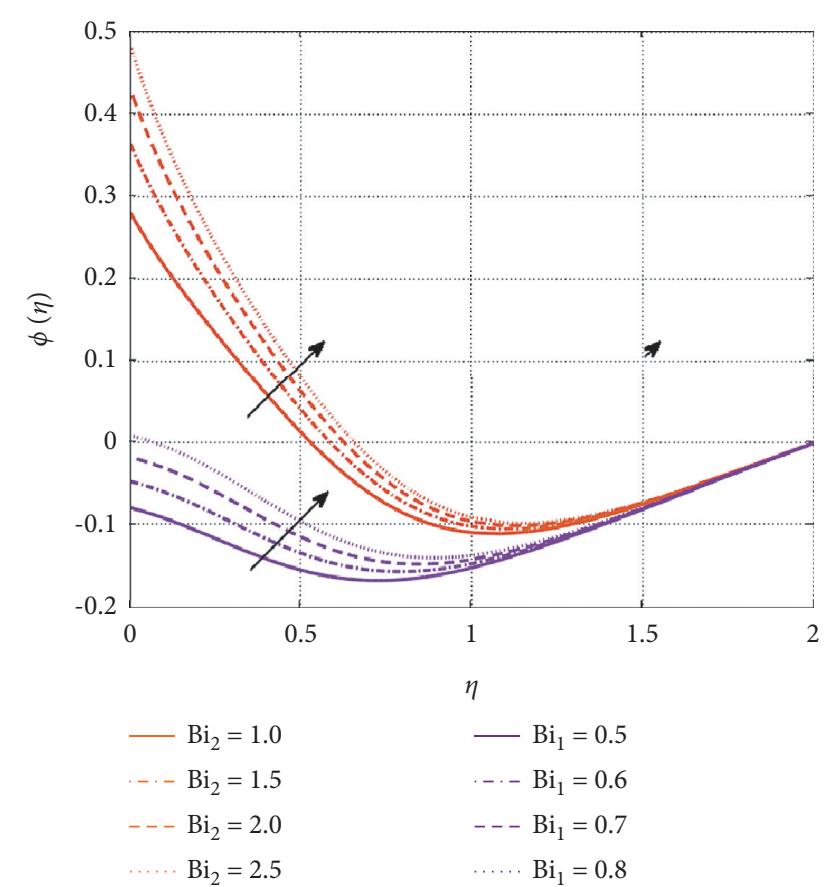

FIGURE 12: Behavior of $\phi(\eta)$ with respect to $\eta$ for $1.0 \leq B i_{1} \leq 2.5$ and $0.5 \leq B i_{2} \leq 0.8$.

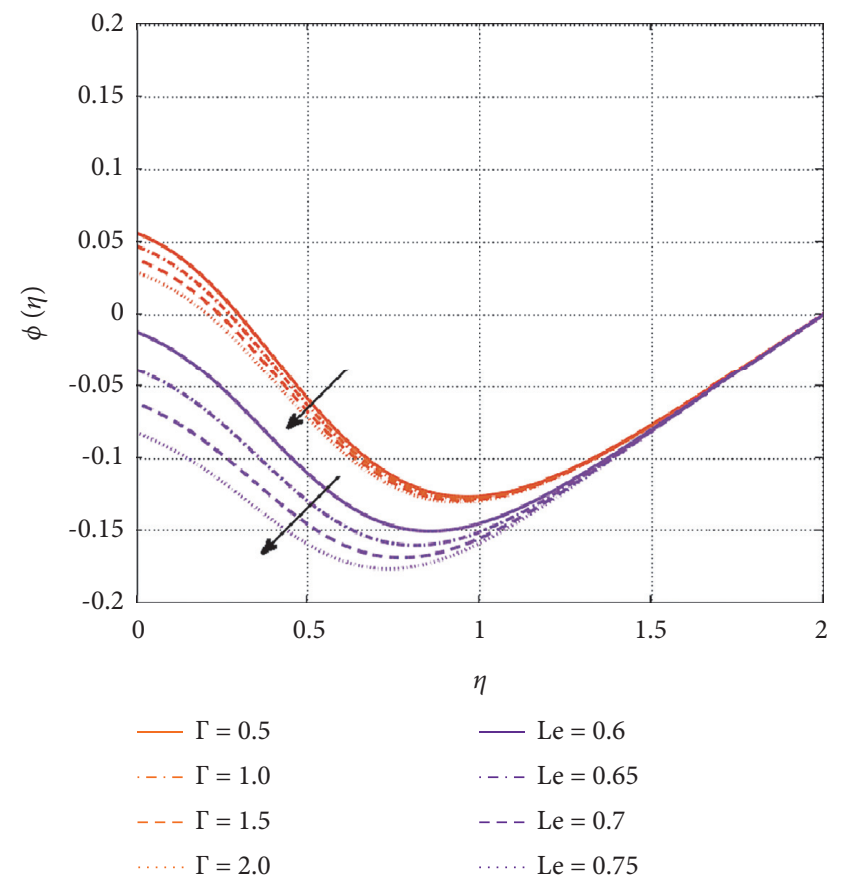

Figure 13: Behavior of $\theta(\eta)$ with respect to $\eta$ for $0.5 \leq \Gamma \leq 2.0$ and $0.6 \leq L e \leq 0.75$.

shows that $\phi(\eta)$ is a diminishing relation of $\Gamma$ and Le. The Lewis number, $L e$, is a dimensional number that is defined as the ratio of thermal diffusivity and mass diffusivity. It has been used to model fluid flows where the heat transfer and mass transfer phenomena are observed to occur simultaneously. In the boundary layer flow, if $L e$ increases, heat diffuses more easily than the solute. Therefore, the thickness of TBL is greater than the thickness of CBL. Note that a reduction in the $\mathrm{CBL}$ reflects a reduction in the concentration profile.

\section{Conclusion}

The considered problem is quite significant in the field of engineering and technology. It has great importance in the production of cosmetics, pharmaceuticals, chemicals, oil, gas, food, and several others. In this study, we investigated the three-dimensional Casson nanofluid flow over a stretching sheet with Arrhenius activation energy and exponential heat source effects with convective heat and mass boundary condition. The numerical solution of the ODEs is obtained using a computational process based on iterative power series (IPS) method combined with shooting iteration approach. The following are some key characteristics of various physical constraints on various flow variables in the problem:

(i) When the magnetic parameter, $M$, increases, the $x-$ and $y$-direction velocities, $f^{\prime}(\eta)$ and $g^{\prime}(\eta)$, decreases. But the rising values of magnetic parameter improve the temperature and concentration distribution.

(ii) The $x$-direction velocity, $f^{\prime}(\eta)$, temperature, $\theta(\eta)$, and concentration, $\phi(\eta)$, are reduced for larger values of stretching ratio parameter, $\alpha$, whereas for the same values of $\alpha$, the $y$-direction velocity, $g^{\prime}(\eta)$, is enhanced.

(iii) The exponential heat source parameter, $Q$, improves the temperature profile. For the concentration profile, we note a decrement initially in the region close to the surface $(0 \leq \eta \leq 0.4)$ and an increment after that.

(iv) Skin friction coefficient is increased by enhancing the Casson parameter, $\beta$, and is decreased for magnetic parameter, $M$, and stretching ratio parameter, $\alpha$.

(v) The strength of the Arrhenius activation energy, E, intensifies the concentration field. Also, enhancement in the thermal and concentration Biot numbers augments the temperature and concentration profiles.

(vi) The heat and mass transfer rates are observed to decline with an increment in the Arrhenius activation energy parameter, $E$.

(vii) The mass transfer rate is enhanced and the heat transfer rate decreased with an increase in the exponential heat source parameter, $Q$.

The results obtained indicate very clearly that thermal and concentration profiles of Casson fluid are enhanced by an exponential heat source and by Arrhenius activation energy. Also, from these concluded points, we have achieved clear-cut answers for the research questions raised in Section 1.

\section{Data Availability}

All the data are available within this article. 


\section{Conflicts of Interest}

The authors declare no conflicts of interest.

\section{Acknowledgments}

The authors would like to acknowledge and express their gratitude to the United Arab Emirates University, Al Ain, UAE, for providing financial support with Grant No. 12 S086.

\section{References}

[1] N. Casson and C. C. Mill, Rheology of Disperse Systems, pp. 84-104, Pergamon Press, Elmsford, NY, USA, 1959.

[2] A. Shafiq, G. Rasool, H. Alotaibi et al., "Thermally enhanced Darcy-Forchheimer Casson-water/glycerine rotating nanofluid flow with uniform magnetic field," Micromachines, vol. 12 , no. 6 , p. $605,2021$.

[3] A. Saeed, Z. Shah, S. Islam et al., "Three-dimensional Casson nanofluid thin film flow over an inclined rotating disk with the impact of heat generation/consumption and thermal radiation," Coatings, vol. 9, no. 4, p. 248, 2019.

[4] M. G. Reddy, P. A. Dinesh, and J. Basavaraj, "3D rotating flow of Casson fluid over an elongated surface with thermophoresis and Brownian moment: Buongiorno model," Journal of Nanofluids, vol. 8, no. 7, pp. 1479-1484, 2019.

[5] P. Durgaprasad, S. V. K. Varma, M. M. Hoque, and C. S. K. Raju, "Combined effects of Brownian motion and thermophoresis parameters on three-dimensional (3D) Casson nanofluid flow across the porous layers slendering sheet in a suspension of graphene nanoparticles," Neural Computing \& Applications, vol. 31, no. 10, pp. 6275-6286, 2019.

[6] P. Durgaprasad, S. Saleem, S. V. K. Varma, and C. S. K. Raju, "Three dimensional slip flow of a chemically reacting Casson fluid flowing over a porous slender sheet with a non-uniform heat source or sink," Journal of the Korean Physical Society, vol. 74, no. 9, pp. 855-864, 2019.

[7] J. Raza, "Thermal radiation and slip effects on magnetohydrodynamic (MHD) stagnation point flow of Casson fluid over a convective stretching sheet," Propulsion and Power Research, vol. 8, no. 2, pp. 138-146, 2019.

[8] T. Thumma, S. R. Mishra, and M. Shamshuddin, "Effect of heat generation and viscous dissipation on MHD 3D Casson nanofluid flow past an impermeable stretching sheet," in Numerical Heat Transfer and Fluid Flow. Lecture Notes in Mechanical Engineering, D. Srinivasacharya and K. Reddy, Eds., Springer, Berlin, Singapore, pp. 575-585, 2019.

[9] M. K. Murthy, S. Sreenadh, P. Lakshminarayana, G. Sucharitha, B. Rushikumar, and J. Nanofluids, "Thermophoresis and Brownian motion effects on three dimensional magnetohydrodynamics slip flow of a Casson nanofluid over an exponentially stretching surface," Journal of Nanofluids, vol. 8, no. 6, pp. 1267-1272, 2019.

[10] L. J. Crane, "Flow past a stretching plate," Zeitschrift für angewandte Mathematik und Physik ZAMP, vol. 21, no. 4, pp. $645-647,1970$.

[11] C. Y. Wang, "The three-dimensional flow due to a stretching flat surface," Physics of Fluids, vol. 27, no. 8, pp. 1915-1917, 1984.

[12] N. Freidoonimehr and A. B. Rahimi, "Brownian motion effect on heat transfer of a three-dimensional nanofluid flow over a stretched sheet with velocity slip," Journal of Thermal Analysis and Calorimetry, vol. 135, no. 1, pp. 207-222, 2019.
[13] M. Hamid, M. Usman, Z. H. Khan, R. Ahmad, and W. Wang, "Dual solutions and stability analysis of flow and heat transfer of Casson fluid over a stretching sheet," Physics Letters A, vol. 383, no. 20, pp. 2400-2408, 2019.

[14] M. Abd El-Aziz and A. A. Afify, "MHD Casson fluid flow over a stretching sheet with entropy generation analysis and Hall influence," Entropy, vol. 21, no. 6, p. 592, 2019.

[15] D. G. Shankar, C. S. K. Raju, M. S. J. Kumar, and O. D. Makinde, "Cattaneo-Christov heat flux on an MHD 3D free convection Casson fluid flow over a stretching sheet," Engineering Transactions, vol. 68, no. 3, pp. 223-238, 2020.

[16] G. Mahanta, M. Das, S. Shaw, and M. L. Mahanta, "Heat and mass transfer of Casson nanofluid flow over a stretching sheet in the presence of magnetic field with Brownian and thermophoretic effects," Journal of Engineering Science \& Technology, vol. 14, no. 5, pp. 3046-3061, 2019.

[17] B. J. Gireesha, M. Archana, B. Mahanthesh, and B. C. Prasannakumara, "Exploration of activation energy and binary chemical reaction effects on nano Casson fluid flow with thermal and exponential space-based heat source," Multidiscipline Modeling in Materials and Structures, vol. 15, no. 1, pp. 227-245, 2019.

[18] T. Hayat, A. Aziz, T. Muhammad, and A. Alsaedi, "Effects of binary chemical reaction and Arrhenius activation energy in Darcy-Forchheimer three-dimensional flow of nanofluid subject to rotating frame," Journal of Thermal Analysis and Calorimetry, vol. 136, no. 4, pp. 1769-1779, 2019.

[19] M. Umar, R. Akhtar, Z. Sabir et al., "Numerical treatment for the three-dimensional Eyring-Powell fluid flow over a stretching sheet with velocity slip and activation energy," Advances in Mathematical Physics, vol. 2019, Article ID 9860471, 2019.

[20] T. Hayat, I. Ullah, M. Waqas, and A. Alsaedi, “Attributes of activation energy and exponential based heat source in flow of Carreau fluid with cross-diffusion effects," Journal of Nonequilibrium Thermodynamics, vol. 44, no. 2, pp. 203-213, 2019.

[21] S. Rashid, T. Hayat, S. Qayyum, M. Ayub, and A. Alsaedi, "Three-dimensional rotating Darcy-Forchheimer flow with activation energy," International Journal of Numerical Methods for Heat \& Fluid Flow, vol. 29, no. 3, pp. 935-948, 2019.

[22] T. Hayat, R. Riaz, A. Aziz, and A. Alsaedi, "Influence of Arrhenius activation energy in MHD flow of third grade nanofluid over a nonlinear stretching surface with convective heat and mass conditions," Physica A: Statistical Mechanics and Its Applications, vol. 549, Article ID 124006, 2020.

[23] S. Rashid, T. Hayat, S. Qayyum, M. Ayub, and A. Alsaedi, "Local similar solutions for flow of an Oldroyd-B nanofluid with activation energy," International Journal of Numerical Methods for Heat \& Fluid Flow, vol. 29, no. 8, pp. 2911-2931, 2019.

[24] S. Rashid, M. I. Khan, T. Hayat, M. Ayub, and A. Alsaedi, "Numerical treatment for rotating Maxwell nanomaterial flow with Arrhenius energy," Applied Nanoscience, vol. 10, no. 8, pp. 2665-2672, 2020.

[25] S. Ahmad, M. Farooq, N. A. Mir, A. Anjum, and M. Javed, "Magneto-hydrodynamic flow of squeezed fluid with binary chemical reaction and activation energy," Journal of Central South University, vol. 26, no. 5, pp. 1362-1373, 2019.

[26] S. Nadeem, R. U. Haq, N. S. Akbar, and Z. H. Khan, "MHD three-dimensional Casson fluid flow past a porous linearly stretching sheet," Alexandria Engineering Journal, vol. 52, no. 4, pp. 577-582, 2013. 
[27] S. Shehzad, T. Hayat, and A. Alsaedi, “Three-dimensional MHD flow of Casson fluid in porous medium with heat generation," Journal of Applied Fluid Mechanics, vol. 9, no. 1, pp. 215-223, 2016.

[28] S. R. Mishra, I. Khan, Q. M. Al-Mdallal, and T. Asifa, "Free convective micropolar fluid flow and heat transfer over a shrinking sheet with heat source," Case Studies in Thermal Engineering, vol. 11, pp. 113-119, 2018.

[29] K. U. Rehman, Q. M. Al-Mdallal, and M. Y. Malik, "Symmetry analysis on thermally magnetized fluid flow regime with heat source/sink," Case Studies in Thermal Engineering, vol. 14, Article ID 100452, 2019.

[30] P. Ragupathi, A. K. A. Hakeem, Q. M. Al-Mdallal, B. Ganga, and S. Saranya, "Non-uniform heat source/sink effects on the three-dimensional flow of $\mathrm{Fe}_{3} \mathrm{O}_{4} / \mathrm{Al}_{2} \mathrm{O}_{3}$ nanoparticles with different base fluids past a Riga plate," Case Studies in Thermal Engineering, vol. 15, Article ID 100521, 2019.

[31] B. Mahanthesh, B. J. Gireesha, B. C. PrasannaKumara, and N. S. Shashikumar, "Marangoni convection radiative flow of dusty nanoliquid with exponential space dependent heat source," Nuclear Engineering and Technology, vol. 49, no. 8, pp. 1660-1668, 2017.

[32] A. Kumar, J. V. R. Reddy, V. Sugunamma, and N. Sandeep, "MHD flow of Carreau fluid over a variable thickness melting surface subject to Cattaneo-Christov heat flux," Multidiscipline Modeling in Materials and Structures, vol. 15, no. 1, pp. 999-1016, 2018.

[33] N. Sandeep and I. L. Animasaun, "Theoretical exploration of exponential heat source and thermal stratification effects on the motion of 3-dimensional flow of Casson fluid over a low heat energy surface at initial unsteady stage," Journal of Theoretical and Applied Mechanics, vol. 47, no. 2, pp. 61-82, 2017.

[34] P. M. Krishna, N. Sandeep, and R. P. Sharma, "Computational analysis of plane and parabolic flow of MHD Carreau fluid with buoyancy and exponential heat source effects," The European Physical Journal-Plus (EPJ Plus), vol. 132, no. 5, pp. 1-15, 2017.

[35] Z. Zia Q, I. Ullah, M. Waqas, A. Alsaedi, and T. Hayat, "Cross diffusion and exponential space dependent heat source impacts in radiated three-dimensional (3D) flow of casson fluid by heated surface," Results Physics, vol. 8, pp. 1275-1282, 2018.

[36] S. Liao and Y. Tan, "A general approach to obtain series solutions of nonlinear differential equations," Studies in Applied Mathematics, vol. 119, no. 4, pp. 297-354, 2007.

[37] R. L. Burden and J. D. Faires, "Numerical Analysis," in Cengage Learning, 1 Brooks/Cole, Pacific Grove, CA, USA, 9th edition, 2021.

[38] U. Al Khawaja and Q. M. Al-Mdallal, "Convergent power series of sech $(\mathrm{x})$ and solutions to nonlinear differential equations," International Journal of Differential Equations, vol. 2018, Article ID 6043936, 2018.

[39] L. Y. Al Sakkaf, Q. M. Al-Mdallal, and U. Al Khawaja, "A numerical algorithm for solving higher-order nonlinear BVPs with an application on fluid flow over a shrinking permeable infinite long cylinder," Complexity, vol. 2018, Article ID 8269541, 2018. 